\title{
Variations in floristic composition of wild and cultivated species associated with Moringa oleifera Lam. in Egypt
}

\author{
Kamal H. Shaltout*, Hussein I. Ali**, Ahmed A. Mobarak***, Dina M. \\ Baraka***and Saadia H. Aly***
}

\begin{abstract}
*Botany Department, Faculty of Science, Tanta University; **Forestry and Wood Technology Department, Faculty of Agriculture, Alexandria University; ***Botany Department, Faculty of Science, Benha University

Corresponding author: Ahmed.moubarak@fsc.bu.edu.eg
\end{abstract}

\section{Abstract}

The aim of this work is to study the cultivated and wild species associated with Moringa oleifera in different sites in Egypt. The cultivated species were 174 related to 145 genera and 67 families, while the wild species were 45 related to 39 genera and 21 families. Fabaceae was the most represented cultivated family (15 species), while Poaceae was the most represented wild family (8 species). Phoenix dactylifera and Portulaca oleracea were the most common cultivated and wild species, respectively. Phanerophytes were the most represented life form,

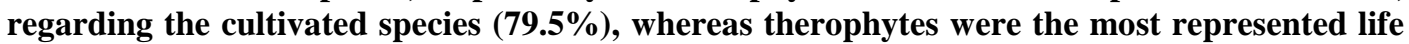
form $(62.2 \%)$. The application of Two Way Indicator Species Analysis (TWINSPAN) on the floristic composition of the sampled stands led to classify them into 7 vegetation groups at level 5 for the cultivated species, and 5 groups at level 3 for the wild species. The cultivated groups were named according to their dominant species as follows: Ficus elastica, Abutilon hirtum, Cordia myxa, Carya illinoensis, Azadirachta indica, Ficus benjamina and Ceiba speciosa. In addition, the wild groups were: Pancratium maritimum, Digitaria ciliaris, Cyperus rotundus, Lantana camara and Bidens pilosa.

Key words: Cultivated species, Wild species, Life form, TWINSPAN, DECORANA.

\section{Introduction}

Moringa oleifera (family: Moringaceae) is native to India and many countries in Africa, Arabia, Southeast Asia and South America (Iqbal \& Bhanger, 2006). This tree is known as "drumstick tree" because its pods are used by drummers, and the "horseradish tree" due to the flavor of its roots (Palada \& Chang, 2003). M. oleifera is a multipurpose that has nutritional (Anwar et al., 2005), medicinal (Kajihara et al., 2008), antifungal (Akinbode \& Ikotun, 2008), antibacterial (Mohan et al., 2006), antiviral (Virmani \& Garg, 2005) and nematicidal properties (Pandey et al., 2001).

In Egypt, Diwan et al. (2004) and Hamdy et al. (2007) listed it as a cultivated species in Orman Botanic Garden. Moreover, Khalifa and Loutfy (2006) recorded it in Faculty of Science and Faculty of Education Botanic Gardens (Ain Shams University),
Zoo Garden plant collection and Aswan Botanic Garden. It was also recorded by Heneidy (2010) in Botanic Garden of Faculty of Science (Alexandria University). Recently, Ammar (2015) listed this tree among the garden flora characterizing the Nile Delta. The aim of this paper was to survey the cultivated and wild species associated with the distribution of $M$. oleifera in many sites in Egypt, and to estimate the main characteristics of these associated species (e.g., life forms, presence and cover percentage).

\section{Material and methods}

Thirteen stands were sampled during September and October 2009 in 8 studied sites in Egypt (Table 1). The coordinates of each stand were determined using GPS. The area of each stand was about $20 \times 20 \mathrm{~m}$, but that of Qanatir Horticulture Research 
Kamal H. Shaltout et al.

Table 1 - Features of the 8 studied botanic sites in Egypt.

\begin{tabular}{|c|c|c|c|c|c|}
\hline Site & Governorate & $\begin{array}{l}\text { Latitude } \\
(\mathrm{N})\end{array}$ & $\begin{array}{l}\text { Longitude } \\
\text { (E) }\end{array}$ & $\begin{array}{l}\text { Area } \\
\text { (ha) }\end{array}$ & $\begin{array}{c}\text { Construction } \\
\text { date }\end{array}$ \\
\hline 1-Sheikh Zuweid Station & North Sinai & $31^{\circ} 14^{\prime} 10^{\prime \prime}$ & $34^{\circ} 06^{\prime} 53^{\prime \prime}$ & 6.9 & 1995 \\
\hline 2- Faculty of Science, Alexandria University & Alexandria & $31^{\circ} 11^{\prime} 23.9^{\prime \prime}$ & $29^{\circ} 54^{\prime} 27.9^{\prime \prime}$ & 1.4 & 1942 \\
\hline 3- Antoniadis Garden & Alexandria & $31^{\circ} 12^{\prime} 15.0^{\prime \prime}$ & $29^{\circ} 56^{\prime} 56.1^{\prime \prime}$ & 18.9 & 1860 \\
\hline 4- Qanatir Horticulture Research Institute & Qalubeiya & $30^{\circ} 10^{\prime} 56.0^{\prime \prime}$ & $31^{\circ} 07^{\prime} 50.7^{\prime \prime}$ & 7.6 & - \\
\hline 5- Faculty of Science $ح$ Ain Shams & Cairo & $30^{\circ} 04^{\prime} 38.7^{\prime \prime}$ & $31^{\circ} 16^{\prime} 56.3^{\prime \prime}$ & 1.3 & 1953 \\
\hline 6- Faculty of Education $\zeta$ University & Cairo & $30^{\circ} 05^{\prime} 29.6^{\prime \prime}$ & $31^{\circ} 18^{\prime} 35.5^{\prime \prime}$ & 0.07 & 1994 \\
\hline 7- Orman Botanic Garden & Giza & $30^{\circ} 01^{\prime} 49.1^{\prime \prime}$ & $31^{\circ} 12^{\prime} 47.1^{\prime \prime}$ & 11.3 & 1873 \\
\hline 8- Aswan Botanic Garden & Aswan & $24^{\circ} 05^{\prime} 33.8^{\prime \prime}$ & $32^{\circ} 53^{\prime} 07.4^{\prime \prime}$ & 7.5 & 1928 \\
\hline
\end{tabular}

Institute was approximately $250 \times 6 \mathrm{~m}$; as the distribution of $M$. oleifera extends along the field edges.

Floristic identification of $M$. oleifera was according to Bailey (1960), while the associated cultivated species in different studied sites were identified according to RHS (1992) and Botanica (2004). The associated wild species were identified according to Täckholm (1974) and Boulos (1999; 2000; 2002; 2005). Moreover, Latin names of species were updated following The Plant List (2013) and APG II System.

In each stand, the presence of cultivated and wild species associated with M. oleifera was recorded (during September and October 2009). Also, the following characters were determined for each species: life form and percent of plant cover. Life forms were identified according to the well-known system of Raunkiaer (1934). The percentage of cover occupied by each associated species was determined visually following BraunBlanquet cover-abundance scale (MullerDombois \& Ellenberg, 1974).

Two trends of multivariate analysis were applied to the vegetation data in the present study: classification and ordination. Both trends have their merits in assisting to understand the vegetation and environmental phenomena. The two-way indicator species analysis, as a classification technique (Hill, 1979 a) and deterended correspondence analysis, as an ordination one (Hill, 1979 b). (TWINSPAN) and (DECORANA) were applied to the matrix of the presence-absence of 219 species (174 cultivated and 45 wild) the designed stands.

\section{Results}

1. Cultivated species

1. 1. Taxonomic diversity
The cultivated species associated with M. oleifera were 174 , related to 145 genera and 67 families (Table 2, Fig. 1). Dicotyledonous families were 49 represented by 111 genera and 134 species; the highly represented of them were Fabacea (15 species), Moraceae ( 9 species), Rutaceae ( 8 species) and Malvaceae (7 species). Each of Apocynaceae, Araliaceae and Solanaceae were represented by 6 species. In addition, 24 dicotyledonous families were represented only by one species. Monocotyledonous families were 12; the highly represented of them were Arecaceae (9 species), Asparagaceae (8 species) and Araceae (5 species). Gymnosperms were represented by 6 families, 8 genera and 8 species.

Each of Cupressaceae and Zamiaceae were represented by 2 species; while each of Araucariaceae, Cycadaceae, Ginkgoaceae and Pinaceae were represented by one species only. Phoenix dactylifera was the most common associated cultivated species (presence $>35 \%$ ), followed by Ailanthus altissima, Duranta erecta, Ficus elastica, Leucaena leucocephala, Olea europaea, Schinus terebinthifolia and Washingtonia robusta.

Taxonomic diversity of the 8 studied sites indicated that the maximum number of species, genera and families was recorded in Faculty of Science - Ain Shams University, while Sheikh Zuweid had the minimum number of species and genera. The maximum number of dicotyledons and monocotyledons was recorded in Botanic Garden of Faculty of Science - Ain Shams University, whereas the minimum number of dicotyledons was recorded in Sheikh Zuweid and that of monocotyledons was recorded in Sheikh Zuweid and Orman Botanic Garden. The 


\section{Kamal H. Shaltout et al.}

maximum number of gymnospermic families was in Faculties of Science and Education -

Ain Sham

University (Table

$3)$.

Table 2 - Cultivated species associated with Moringa oleifera in the 13 studied stands in Egypt. P: presence and $\mathrm{C}$ : cover. Life forms are coded as follows: $\mathrm{Ph}$ : phanerophytes, $\mathrm{Ch}$ : chamaephytes, Hc: hemicryptophytes, Ge: geophytes, Th: therophytes.

\begin{tabular}{|c|c|c|c|c|}
\hline \multirow{2}{*}{ Species } & \multirow{2}{*}{ Family } & \multirow{2}{*}{ Life form } & $\mathrm{P}$ & C \\
\hline & & & \multicolumn{2}{|c|}{\begin{tabular}{l|l}
$P$ & $C$ \\
$(\%)$
\end{tabular}} \\
\hline Abutilon hirtum (Lam.) Sweet. & Malvaceae & $\mathrm{Ch}$ & 28.6 & 15 \\
\hline Acacia farnesiana (L.) Willd. & Fabaceae & $\mathrm{Ph}$ & 7.1 & 1 \\
\hline Acalypha wilkesiana Müell. Arg. & Euphorbiaceae & $\mathrm{Ph}$ & 14.3 & 2 \\
\hline Acer negundo L. & Sapindaceae & $\mathrm{Ph}$ & 7.1 & 4 \\
\hline $\begin{array}{l}\text { Acokanthera oblongifolia (Hochst.) Benth. \& } \\
\text { Hook.f. ex B. D. Jacks. }\end{array}$ & Apocynaceae & $\mathrm{Ph}$ & 14.3 & 4 \\
\hline Agave americana $\mathrm{L}$. & Asparagaceae & $\mathrm{Hc}$ & 14.3 & 3 \\
\hline Ailanthus altissima (Mill.) Swingle & Simaroubaceae & $\mathrm{Ph}$ & 35.7 & 11 \\
\hline Alcea rosea $\mathrm{L}$. & Malvaceae & $\mathrm{Ch}$ & 14.3 & 3 \\
\hline Alocasia macrorrhizos (L.) G. Don & Araceae & $\mathrm{Ch}$ & 14.3 & 2 \\
\hline Aloe ciliaris Haw. & Xanthorrhoeaceae & $\mathrm{Hc}$ & 7.1 & 1 \\
\hline A. vera (L.) Brum. f. & Xanthorrhoeaceae & $\mathrm{Hc}$ & 7.1 & 1 \\
\hline Alpinia officinarum Hance & Zingiberaceae & $\mathrm{Ge}$ & 7.1 & 1 \\
\hline Annona muricata $\mathrm{L}$. & Annonaceae & $\mathrm{Ph}$ & 7.1 & 1 \\
\hline A. squamosa $\mathrm{L}$. & Annonaceae & $\mathrm{Ph}$ & 7.1 & 1 \\
\hline Araucaria heterophylla (Salisb.) Franco & Araucariaceae & $\mathrm{Ph}$ & 14.3 & 4 \\
\hline Aristolochia littoralis Parodi & Aristolochiaceae & $\mathrm{Hc}$ & 7.1 & 1 \\
\hline Asparagus densiflorus (Kunth) Jessop & Asparagaceae & $\mathrm{Hc}$ & 7.1 & 1 \\
\hline A. setaceus (Kunth) Jessop & Asparagaceae & $\mathrm{Hc}$ & 7.1 & 1 \\
\hline Azadirachta indica A Juss. & Meliaceae & $\mathrm{Ph}$ & 14.3 & 11 \\
\hline Bauhinia variegata $\mathrm{L}$. & Fabaceae & $\mathrm{Ph}$ & 7.1 & 4 \\
\hline Beaumontia grandiflora Wall. & Apocynaceae & $\mathrm{Ph}$ & 14.3 & 3 \\
\hline Bougainvillea spectabilis Willd. & Nyctaginaceae & $\mathrm{Ph}$ & 7.1 & 1 \\
\hline $\begin{array}{l}\text { Brachychiton acerifolius (A. Cunn. ex G. Don) F. } \\
\text { Muell. }\end{array}$ & Malvaceae & $\mathrm{Ph}$ & 7.1 & 1 \\
\hline Breynia disticha J. R. Forst. \& G. Forst. & Phyllanthaceae & $\mathrm{Ph}$ & 7.1 & 1 \\
\hline Brunfelsia australis Benth. & Solanaceae & $\mathrm{Ph}$ & 7.1 & 1 \\
\hline Caesalpinia palmeri S. Watson & Fabaceae & $\mathrm{Ph}$ & 7.1 & 2 \\
\hline Canna indica $\mathrm{L}$. & Cannaceae & $\mathrm{Ge}$ & 7.1 & 1 \\
\hline Cardiospermum halicacabum L. & Sapindaceae & $\mathrm{Ph}$ & 7.1 & 2 \\
\hline Carya illinoensis (Wangenh.) K. Koch & Juglandaceae & $\mathrm{Ph}$ & 21.4 & 19 \\
\hline Caryota mitis Lour. & Arecaceae & $\mathrm{Ph}$ & 7.1 & 1 \\
\hline Cascabela thevetia (L.) Lippold & Apocynaceae & $\mathrm{Ph}$ & 21.4 & 6 \\
\hline Casimiroa edulis La Llave & Rutaceae & $\mathrm{Ph}$ & 14.3 & 2 \\
\hline Cassia fistula $\mathrm{L}$. & Fabaceae & $\mathrm{Ph}$ & 7.1 & 1 \\
\hline Ceiba speciosa (A. St. - Hil.) Ravenna & Malvacea & $\mathrm{Ph}$ & 21.4 & 17 \\
\hline Cereus uruguayanus R. Kiesling & Cactaceae & $\mathrm{Ph}$ & 7.1 & 1 \\
\hline Cestrum nocturnum L. & Solanaceae & $\mathrm{Ph}$ & 14.3 & 4 \\
\hline C. parqui (Lam.) L'Hér. & Solanaceae & $\mathrm{Ph}$ & 7.1 & 1 \\
\hline Cinnamomum verum J. Presl & Lauraceae & $\mathrm{Ph}$ & 14.3 & 2 \\
\hline Citharexylum spinosum L. & Verbenaceae & $\mathrm{Ph}$ & 7.1 & 2 \\
\hline Citrus aurantium L. & Rutaceae & $\mathrm{Ph}$ & 14.3 & 2 \\
\hline C. aurantifolia (Christm.) Swingle & Rutaceae & $\mathrm{Ph}$ & 7.1 & 1 \\
\hline
\end{tabular}


Kamal H. Shaltout et al.

Table 2 - cont. 1

\begin{tabular}{|c|c|c|c|c|}
\hline \multirow{2}{*}{ Species } & \multirow{2}{*}{ Family } & \multirow{2}{*}{ Life form } & $\mathrm{P}$ & $\mathrm{C}$ \\
\hline & & & \multicolumn{2}{|c|}{$(\%)$} \\
\hline C. maxima (Brum.) Merr. & Rutaceae & $\mathrm{Ph}$ & 7.1 & 2 \\
\hline C. reticulata Blanco & Rutaceae & $\mathrm{Ph}$ & 7.1 & 1 \\
\hline C. sinensis (L.) Osbeck. & Rutaceae & $\mathrm{Ph}$ & 14.3 & 2 \\
\hline Clematis flammula $\mathrm{L}$. & Ranunculaceae & $\mathrm{Ph}$ & 7.1 & 2 \\
\hline Clivia miniata (Lindl.) Boose & Amaryllidaceae & $\mathrm{Hc}$ & 7.1 & 2 \\
\hline Codiaeum variegatum (L.) Rumph. ex A. Juss. & Euphorbiaceae & $\mathrm{Ph}$ & 28.6 & 4 \\
\hline Coffea arabica $\mathrm{L}$ & Rubiaceae & $\mathrm{Ph}$ & 7.1 & 1 \\
\hline Cordia myxa L. & Boraginaceae & $\mathrm{Ph}$ & 7.1 & 7 \\
\hline Cupressus sempervirens L. & Cupressaceae & $\mathrm{Ph}$ & 14.3 & 3 \\
\hline Cycas revoluta Thunb. & Cycadaceae & $\mathrm{Ph}$ & 21.4 & 3 \\
\hline Cyperus alternifolius L. & Cyperaceae & $\mathrm{Hc}$ & 14.3 & 3 \\
\hline Dendrocalamus giganteus Munro & Poaceae & $\mathrm{Ph}$ & 14.3 & 11 \\
\hline Dichrostachys cinerea (L.) Wight \& Arn. & Fabaceae & $\mathrm{Ph}$ & 7.1 & 1 \\
\hline Dioon edule Lind. & Zamiaceae & $\mathrm{Ph}$ & 7.1 & 2 \\
\hline Dodonaea viscosa (L.) Jacq. & Sapindaceae & $\mathrm{Ph}$ & 21.4 & 2 \\
\hline Dombeya burgessiae Gerr. ex Harv. & Malvaceae & $\mathrm{Ph}$ & 14.3 & 4 \\
\hline Dracaena marginata hort. & Asparagaceae & $\mathrm{Hc}$ & 28.6 & 11 \\
\hline Duranta erecta $\mathrm{L}$. & Verbenaceae & $\mathrm{Ph}$ & 35.7 & 7 \\
\hline Enterolobium cyclocarpum (Jacq.) Griseb. & Fabaceae & $\mathrm{Ph}$ & 7.1 & 3 \\
\hline Eriobotrya japonica (Thunb.) Lindl. & Rosaceae & $\mathrm{Ph}$ & 21.4 & 3 \\
\hline Erythrina caffra Thunb. & Fabaceae & $\mathrm{Ph}$ & 7.1 & 2 \\
\hline E.corallodendrum L. & Fabaceae & $\mathrm{Ph}$ & 7.1 & 2 \\
\hline Eugenia uniflora $\mathrm{L}$ & Myrtaceae & $\mathrm{Ph}$ & 7.1 & 1 \\
\hline Euonymus japonicus Thunb. & Celasteraceae & $\mathrm{Ph}$ & 7.1 & 1 \\
\hline Ficus benjamina $\mathrm{L}$. & Moraceae & $\mathrm{Ph}$ & 21.4 & 9 \\
\hline F. carica $\mathrm{L}$. & Moraceae & $\mathrm{Ph}$ & 7.1 & 2 \\
\hline F. deltoidea Jack & Moraceae & $\mathrm{Ph}$ & 7.1 & 3 \\
\hline F. elastica Roxb. ex Hornem. & Moraceae & $\mathrm{Ph}$ & 35.7 & 14 \\
\hline F. lyrata Warb. & Moraceae & $\mathrm{Ph}$ & 14.3 & 5 \\
\hline F. microcarpa L. f. & Moraceae & $\mathrm{Ph}$ & 21.4 & 6 \\
\hline F. sycomorus L. & Moraceae & $\mathrm{Ph}$ & 7.1 & 6 \\
\hline F. virens Aiton & Moraceae & $\mathrm{Ph}$ & 7.1 & 3 \\
\hline Firmiana simplex (L.) W. Wight & Malvaceae & $\mathrm{Ph}$ & 7.1 & 1 \\
\hline Galium sp. & Rubiaceae & $\mathrm{Ch}$ & 7.1 & 1 \\
\hline Gerbera jamesonii Bolus ex Hook. f. & Asteraceae & $\mathrm{Hc}$ & 7.1 & 1 \\
\hline Ginkgo biloba $\mathrm{L}$ & Ginkgoaceae & $\mathrm{Ph}$ & 14.3 & 2 \\
\hline Haematoxylum campechianum L. & Fabaceae & $\mathrm{Ph}$ & 14.3 & 2 \\
\hline Harpullia pendula Planch. ex F. Muell. & Sapindaceae & $\mathrm{Ph}$ & 7.1 & 3 \\
\hline Hedera helix $\mathrm{L}$. & Araliaceae & $\mathrm{Ph}$ & 7.1 & 2 \\
\hline Hemerocallis fulva (L.) L. & Xanthorrhoeaceae & $\mathrm{Ge}$ & 7.1 & 1 \\
\hline Hibiscus rosa-sinensis $\mathrm{L}$. & Malvaceae & $\mathrm{Ph}$ & 14.3 & 3 \\
\hline Hiptage sp. & Malpighiaceae & $\mathrm{Ph}$ & 7.1 & 1 \\
\hline Hyphaene thebaica (L.) Mart. & Arecaceae & $\mathrm{Ph}$ & 7.1 & 1 \\
\hline Ipomoea cairica (L.) Sweet & Convolvulaceae & $\mathrm{Hc}$ & 7.1 & 2 \\
\hline
\end{tabular}


Kamal H. Shaltout et al.

Table 2 - cont. 2

\begin{tabular}{|c|c|c|c|c|}
\hline \multirow{2}{*}{ Species } & \multirow{2}{*}{ Family } & \multirow{2}{*}{ Life form } & $\mathrm{P}$ & $\mathrm{C}$ \\
\hline & & & \multicolumn{2}{|l|}{$(\%)$} \\
\hline I. carnea Jacq. & Convolvulaceae & $\mathrm{Ch}$ & 14.3 & 3 \\
\hline Jacaranda mimosifolia D. Don & Bignoniaceae & $\mathrm{Ph}$ & 7.1 & 2 \\
\hline Jasminum officinale L. & Oleaceae & $\mathrm{Ph}$ & 7.1 & 1 \\
\hline Jatropha curcas L. & Euphorbiaceae & $\mathrm{Ph}$ & 7.1 & 2 \\
\hline Justicia adhatoda $\mathrm{L}$. & Acanthaceae & $\mathrm{Ph}$ & 14.3 & 4 \\
\hline Kigelia africana (Lam.) Benth. & Bignoniaceae & $\mathrm{Ph}$ & 7.1 & 2 \\
\hline Koelreuteria paniculata Laxm. & Sapindaceae & $\mathrm{Ph}$ & 14.3 & 2 \\
\hline Lagerstroemia indica $\mathrm{L}$. & Lythraceae & $\mathrm{Ph}$ & 7.1 & 1 \\
\hline Laurus nobilis L. & Lauraceae & $\mathrm{Ph}$ & 28.6 & 8 \\
\hline Lawsonia inermis $\mathrm{L}$. & Lythraceae & $\mathrm{Ph}$ & 7.1 & 1 \\
\hline Leucaena leucocephala (Lam.) De Wit. & Fabaceae & $\mathrm{Ph}$ & 35.7 & 14 \\
\hline Livistona chinensis (Jacq.) R. Br. ex Mart. & Arecaceae & $\mathrm{Ph}$ & 7.1 & 2 \\
\hline Lonicera japonica Thunb. & Caprifoliaceae & $\mathrm{Ch}$ & 7.1 & 2 \\
\hline Lycianthes rantonnei (Carrière) Bitter & Solanaceae & $\mathrm{Ph}$ & 14.3 & 2 \\
\hline Magnolia grandiflora $\mathrm{L}$. & Magnoliaceae & $\mathrm{Ph}$ & 7.1 & 2 \\
\hline Mangifera indica $\mathrm{L}$. & Anacardiaceae & $\mathrm{Ph}$ & 7.1 & 2 \\
\hline Markhamia lutea (Benth.) K. Schum. & Bignoniaceae & $\mathrm{Ph}$ & 7.1 & 2 \\
\hline Melaleuca ericifolia $\mathrm{Sm}$. & Myrtaceae & $\mathrm{Ph}$ & 7.1 & 2 \\
\hline Mesembryanthemum cordifolium L. f. & Aizoaceae & $\mathrm{Ch}$ & 7.1 & 1 \\
\hline Monstera deliciosa Liebm. & Araceae & $\mathrm{Ph}$ & 14.3 & 3 \\
\hline Morus alba $\mathrm{L}$. & Moraceae & $\mathrm{Ph}$ & 14.3 & 2 \\
\hline Murraea paniculata (L.) Jack & Rutaceae & $\mathrm{Ph}$ & 14.3 & 2 \\
\hline Musa paradisiaca $\mathrm{L}$. & Musaceae & $\mathrm{Ph}$ & 14.3 & 2 \\
\hline Nandina domestica Thunb. & Berberidaceae & $\mathrm{Ph}$ & 7.1 & 1 \\
\hline Nephthytis afzelii Schott. & Araceae & $\mathrm{Hc}$ & 21.4 & 3 \\
\hline Nerium oleander L. & Apocynaceae & $\mathrm{Ph}$ & 21.4 & 4 \\
\hline Ocimum basilicum L. & Lamiaceae & $\mathrm{Ch}$ & 7.1 & 1 \\
\hline Olea europaea $\mathrm{L}$. & Oleaceae & $\mathrm{Ph}$ & 35.7 & 10 \\
\hline Oreopanax reticulatum Willd & Araliaceae & $\mathrm{Ph}$ & 7.1 & 1 \\
\hline Passiflora caerulea L. & Passifloraceae & $\mathrm{Ph}$ & 14.3 & 2 \\
\hline Pelarogonium zonale (L.) L'Hér. ex Aiton & Geraniaceae & $\mathrm{Ph}$ & 7.1 & 1 \\
\hline Persea Americana Mill. & Lauraceae & $\mathrm{Ph}$ & 7.1 & 1 \\
\hline Phoenix canariensis Hort. ex Chabaud. & Arecaceae & $\mathrm{Ph}$ & 7.1 & 2 \\
\hline Ph. dactylifera $\mathrm{L}$. & Arecaceae & $\mathrm{Ph}$ & 50 & 25 \\
\hline Phytolacca dioica $\mathrm{L}$. & Phytolaccaceae & $\mathrm{Ph}$ & 14.3 & 5 \\
\hline Pinus sp. & Pinaceae & $\mathrm{Ph}$ & 7.1 & 1 \\
\hline Pleiogynium timoriense (DC.) leenth. & Anacardiacea & $\mathrm{Ph}$ & 7.1 & 1 \\
\hline Plinia edulis (Vell.) Sobral & Myrtaceae & $\mathrm{Ph}$ & 7.1 & 1 \\
\hline Plumeria alba H. B. \& K. & Apocynaceae & $\mathrm{Ph}$ & 7.1 & 1 \\
\hline Polyscias guilfoylei (W. Bull) L. H. Bail. & Araliaceae & $\mathrm{Ph}$ & 7.1 & 2 \\
\hline Pongamia pinnata (L.) Merr. & Fabaceae & $\mathrm{Ph}$ & 7.1 & 2 \\
\hline Poplus nigra $\mathrm{L}$. & Salicaceae & $\mathrm{Ph}$ & 7.1 & 2 \\
\hline Prunus persica L. & Rosaceae & $\mathrm{Ph}$ & 7.1 & 1 \\
\hline Punica granatum $\mathrm{L}$. & Punicaceae & $\mathrm{Ph}$ & 7.1 & 1 \\
\hline
\end{tabular}


Kamal H. Shaltout et al.

Table 2 - cont. 3

\begin{tabular}{|c|c|c|c|c|}
\hline \multirow{2}{*}{ Species } & \multirow{2}{*}{ Family } & \multirow{2}{*}{ Life form } & $\mathrm{P}$ & $\mathrm{C}$ \\
\hline & & & \multicolumn{2}{|c|}{$(\%)$} \\
\hline Quercus robur L. & Fagaceae & $\mathrm{Ph}$ & 7.1 & 6 \\
\hline Rosa sp. & Rosaceae & $\mathrm{Ph}$ & 28.6 & 5 \\
\hline Rosmarinus officinalis L. & Lamiaceae & $\mathrm{Ph}$ & 7.1 & 1 \\
\hline Roystonea regia (Kunth) O. F. Cook & Arecaceae & $\mathrm{Ph}$ & 14.3 & 4 \\
\hline Ruscus aculeatus L. & Asparagaceae & $\mathrm{Ge}$ & 28.6 & 4 \\
\hline Ruta graveolens $\mathrm{L}$. & Rutaceae & $\mathrm{Ph}$ & 14.3 & 2 \\
\hline $\begin{array}{l}\text { Sabal Palmetto (Walt.) Lodd. ex Schult. \& } \\
\text { Schult. f. }\end{array}$ & Arecaceae & $\mathrm{Ph}$ & 14.3 & 2 \\
\hline Salix mucronata Thunb. & Salicaceae & $\mathrm{Ph}$ & 7.1 & 1 \\
\hline Salvia splendes Sellow ex Schult. & Lamiaceae & $\mathrm{Ch}$ & 7.1 & 1 \\
\hline Sambucus nigra L. & Adoxaceae & $\mathrm{Ph}$ & 14.3 & 2 \\
\hline Sansevieria trifasciata Prain & Asparagaceae & $\mathrm{Ge}$ & 7.1 & 1 \\
\hline Schefflera actinophylla (Endl.) Harms & Araliaceae & $\mathrm{Ph}$ & 7.1 & 2 \\
\hline $\begin{array}{l}\text { S. elegantissima (Veitch ex Mast.) Lowry \& } \\
\text { Frodin }\end{array}$ & Araliaceae & $\mathrm{Ph}$ & 7.1 & 3 \\
\hline S. umbellifera (Sond.) Baill. & Araliaceae & $\mathrm{Ph}$ & 14.3 & 4 \\
\hline Schinus terebinthifolia Raddi & Anacardiaceae & $\mathrm{Ph}$ & 35.7 & 6 \\
\hline Schotia brachypetala Sond. & Fabaceae & $\mathrm{Ph}$ & 7.1 & 1 \\
\hline $\begin{array}{l}\text { Senna didymobotrya (Fres.) H. S. Irwin \& } \\
\text { Barneby }\end{array}$ & Fabaceae & $\mathrm{Ph}$ & 7.1 & 1 \\
\hline S. occidentalis (L.) Link & Fabaceae & $\mathrm{Th}$ & 7.1 & 1 \\
\hline $\begin{array}{l}\text { Simmondsia chinensis (Link) C. K. } \\
\text { Schneid. }\end{array}$ & Simmondsiaceae & $\mathrm{Ph}$ & 7.1 & 1 \\
\hline Solandra grandiflora $\mathrm{Sw}$ & Solanaceae & $\mathrm{Ph}$ & 7.1 & 2 \\
\hline Solanum diphyllum $\mathrm{L}$. & Solanaceae & $\mathrm{Ph}$ & 21.4 & 3 \\
\hline Sophora secundiflora (Ortega) DC. & Fabaceae & $\mathrm{Ph}$ & 14.3 & 2 \\
\hline Spiraea thunbergii Siebold ex Blume & Rosaceae & $\mathrm{Ph}$ & 7.1 & 1 \\
\hline Strelitzia nicoli Regel. \& K. Koch & Strelitziaceae & $\mathrm{Ph}$ & 14.3 & 2 \\
\hline S. reginae Banks & Strelitziaceae & $\mathrm{Hc}$ & 7.1 & 1 \\
\hline $\begin{array}{l}\text { Symphyotrichum novi-belgii (L.) G. L. } \\
\text { Nesom }\end{array}$ & Asteraceae & $\mathrm{Hc}$ & 7.1 & 1 \\
\hline Syngonium podophyllum Schott & Araceae & $\mathrm{Hc}$ & 14.3 & 2 \\
\hline Tabebuia rosea (Betrol.) Bertero ex A. DC. & Bignoniaceae & $\mathrm{Ph}$ & 7.1 & 1 \\
\hline $\begin{array}{l}\text { Tabernaemontana divaricata (L.) R. Br. ex } \\
\text { Roem. \& Schult. }\end{array}$ & Apocynaceae & $\mathrm{Ph}$ & 14.3 & 2 \\
\hline Taxodium distichum (L.) Rich. & Cupressaceae & $\mathrm{Ph}$ & 14.3 & 5 \\
\hline Terminalia catappa $\mathrm{L}$. & Combretaceae & $\mathrm{Ph}$ & 7.1 & 2 \\
\hline T. arjuna (Roxb. ex DC.) Wight \& Arn. & Combretaceae & $\mathrm{Ph}$ & 7.1 & 4 \\
\hline T. mulleri Benth. & Combretaceae & $\mathrm{Ph}$ & 7.1 & 1 \\
\hline Tradescantia pallida (Rose) D. R. Hunt & Commelinaceae & $\mathrm{Ch}$ & 7.1 & 1 \\
\hline Vitex agnus-castus $\mathrm{L}$. & Lamiaceae & $\mathrm{Ch}$ & 14.3 & 2 \\
\hline Vitis vinifera $\mathrm{L}$. & Vitaceae & $\mathrm{Ph}$ & 14.3 & 2 \\
\hline Volkameria inermis L. & Lamiaceae & $\mathrm{Ph}$ & 7.1 & 1 \\
\hline $\begin{array}{l}\text { Washingtonia filifera (Linden ex André) } \\
\text { H.Wendl. ex de Bary }\end{array}$ & Arecaceae & $\mathrm{Ph}$ & 7.1 & 2 \\
\hline W. robusta $\mathrm{H}$. Wendl. & Arecaceae & $\mathrm{Ph}$ & 35.7 & 10 \\
\hline Yucca aloifolia L. & Asparagaceae & $\mathrm{Ph}$ & 7.1 & 1 \\
\hline
\end{tabular}


Kamal H. Shaltout et al.

\begin{tabular}{|c|c|c|c|c|}
\hline \multirow{2}{*}{ Species } & \multirow{2}{*}{ Family } & \multirow{2}{*}{ Life form } & $\mathrm{P}$ & $\mathrm{C}$ \\
\hline & & & \multicolumn{2}{|l|}{$(\%)$} \\
\hline Yucca gigantea Lem. & Asparagaceae & $\mathrm{Ph}$ & 14.3 & 2 \\
\hline Zamia furfurasea L. f. ex Aiton & Zamiaceae & $\mathrm{Hc}$ & 7.1 & 1 \\
\hline Zantedeschia aethiopica (L.) Spreng. & Araceae & $\mathrm{Ge}$ & 7.1 & 1 \\
\hline Ziziphus jujuba Mill. & Rhamnaceae & $\mathrm{Ph}$ & 7.1 & 1 \\
\hline Z. spina-christi (L.) Desf. & Rhamnaceae & $\mathrm{Ph}$ & 14.3 & 2 \\
\hline
\end{tabular}

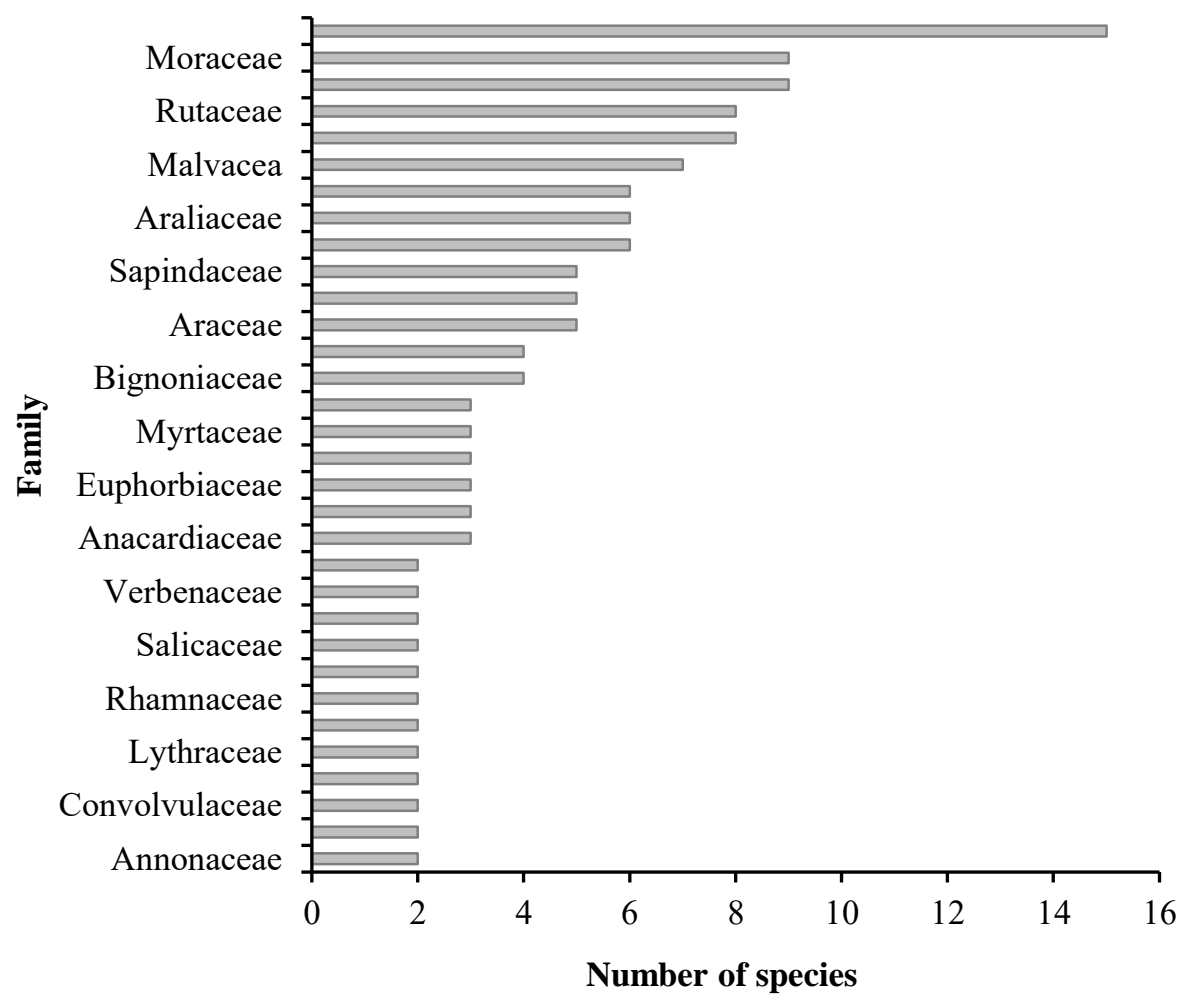

Fig. 1- Number of cultivated species associated with Moringa oleifera in relation to their families (families of $<2$ species were not represented).

\section{2. Life forms}

Determination of the life forms of the cultivated species associated with $M$. oleifera indicated that the most represented life form was the phanerophytes ( 140 species $=79.5 \%$ of the total species) followed by the hemicryptophytes $(16$ species $=9.1 \%$ of the total species), then chamaephytes (11 species $=6.3 \%$ of the total species) and geophytes ( 6 species $=3.4 \%$ of the total species). On the other hand, therophytes were the least represented (Fig. 2). Regarding the flora of the 8 studied sites, phanerophytes had the maximum representation in Faculty of
Science - Ain Shams University, and the minimum in Sheikh Zuweid and Aswan Botanic Garden. Chamaephytes had the highest representation in Faculty of Science Ain Shams University, while thus absent in Sheikh Zuweid and Orman Botanic Garden. Moreover, hemicryptophytes and geophytes were recorded in 3 studied sites only and their maximum representation was in Faculty of Science - Ain Shams University and Faculty of Science - Alexandria University. Therophytes were restricted to Faculty of Science - Ain Shams University (Table 4). 



\section{Kamal H. Shaltout et al.}

Table 3 - Taxonomic diversity of the cultivated species associated with Moringa oleifera in 8 studied sites in Egypt. Sheikh Zuweid Station (SZ), Botanic Garden of Faculty of Science Alexandria University (XS), Antoniadis Botanic Garden (XA), Qanatir Horticulture Research Institute (QA), Botanic Garden of Faculty of Science - Ain Shams University (SA), Botanic Garden of Faculty of Education - Ain Shams University (ED), Orman Botanic Garden (OR) and Aswan Botanic Garden (AS).

\begin{tabular}{|l|rrrrrrrr|r|}
\hline \multirow{2}{*}{ Taxic variable } & \multicolumn{7}{|c|}{ Study site } & \multirow{2}{*}{ Total } \\
\cline { 2 - 7 } Dicotyledons & SZ & XS & XA & QA & SA & ED & OR & AS & \\
Species & 3 & 23 & 14 & 6 & 80 & 49 & 10 & 4 & 134 \\
Genus & 3 & 23 & 14 & 5 & 66 & 47 & 10 & 4 & 111 \\
Family & 3 & 15 & 14 & 5 & 38 & 26 & 7 & 4 & 49 \\
Monocotyledons & & & & & & & & & \\
Species & 1 & 11 & 3 & 3 & 19 & 8 & 1 & 2 & 32 \\
Genus & 1 & 10 & 3 & 3 & 16 & 8 & 1 & 2 & 26 \\
Family & 1 & 4 & 1 & 2 & 8 & 5 & 1 & 1 & 12 \\
Gymnosperms & & & & & & & & & \\
Species & 1 & 2 & 1 & - & 4 & 5 & - & - & 8 \\
Genus & 1 & 2 & 1 & - & 4 & 5 & - & - & 8 \\
Family & 1 & 2 & 1 & - & 4 & 4 & - & - & 6 \\
\hline Total & & & & & & & & & \\
Species & 5 & 36 & 18 & 9 & 103 & 62 & 11 & 6 & 174 \\
Genus & 5 & 35 & 18 & 8 & 86 & 60 & 11 & 6 & 145 \\
Family & 5 & 21 & 16 & 7 & 50 & 35 & 8 & 5 & 67 \\
\hline
\end{tabular}

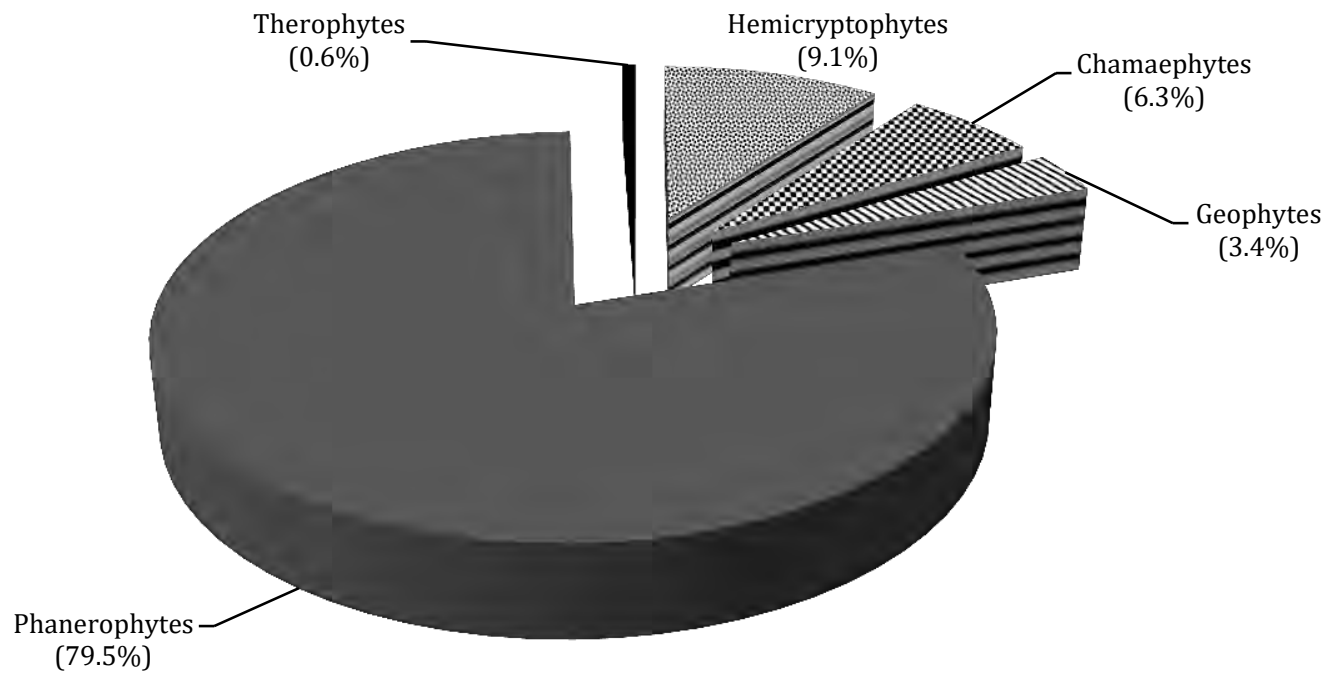

Fig. 2 - Life form spectrum of the cultivated species associated with Moringa oleifera in 8 studied sites in Egypt. 


\section{Kamal H. Shaltout et al.}

Table 4 - Life form spectrum of the cultivated species associated with Moringa oleifera in 8 studied sites in Egypt: Sheikh Zuweid Station (SZ), Botanic Garden of Faculty of Science Alexandria University (XS), Antoniadis Botanic Garden (XA), Qanatir Horticulture Research Institute (QA), Botanic Garden of Faculty of Science - Ain Shams University (SA), Botanic Garden of Faculty of Education - Ain Shams University (ED), Orman Botanic Garden (OR) and Aswan Botanic Garden (AS).

\begin{tabular}{|l|rrrrrrrr|r|}
\hline \multirow{2}{*}{ Life form } & \multicolumn{7}{|c|}{ Study site } & \multirow{2}{*}{ Total } \\
\cline { 2 - 9 } & SZ & XS & XA & QA & SA & ED & OR & AS & \\
\hline Phanerophytes & 5 & 23 & 17 & 8 & 85 & 57 & 11 & 5 & 140 \\
Chamaephytes & - & 4 & 1 & 1 & 6 & 1 & - & 1 & 11 \\
Hemicryptophytes & - & 4 & - & - & 10 & 3 & - & - & 16 \\
Geophytes & - & 5 & - & - & 1 & 1 & - & - & 6 \\
Therophytes & - & - & - & - & 1 & - & - & - & 1 \\
\hline Total & 5 & 36 & 18 & 9 & 103 & 62 & 11 & 6 & 174 \\
\hline
\end{tabular}

\subsection{Vegetation analysis}

The application of Two Way Indicator Species Analysis (TWINSPAN) on the floristic composition of the 13 sampled stands led to classify them into 7 vegetation groups at level 5 (Fig. 3 A). The application of DECORANA on the same set of data indicates a reasonable segregation between these groups along the ordination axes 1 and 2 (Fig. 3 B). Four groups contained only one stand: Group I (Orman Botanic Garden) and dominated by Ficus elastica. Group II (Faculty of Science - Ain Shams University) and dominated by Abutilon hirtum, group III (Faculty of Science - Ain Shams University) and dominated by Cordia myxa and group VI (Antoniadis Garden) and dominated by Ficus benjamina. Group IV consisted of 2 stands (Sheikh Zuweid and Faculty of Science - Ain Shams University) and dominated by Carya illinoensis, while group $\mathbf{V}$ included 4 stands (2 stands in Sheikh Zuweid, and one stand in each of Qanatir Horticulture Research Institute and Aswan Botanic Garden) and dominated by Azadirachta indica and group VII

\section{A- TWINSPAN classification}

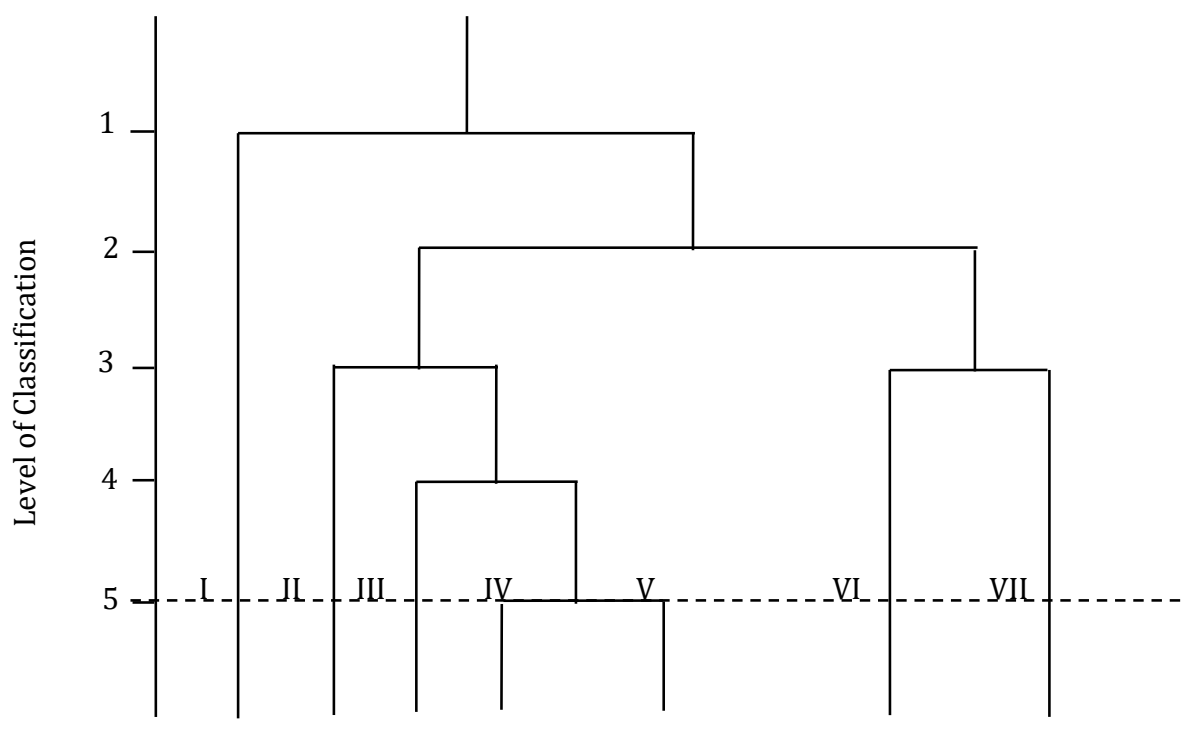




\section{Kamal H. Shaltout et al.}

B- DECORANA ordination

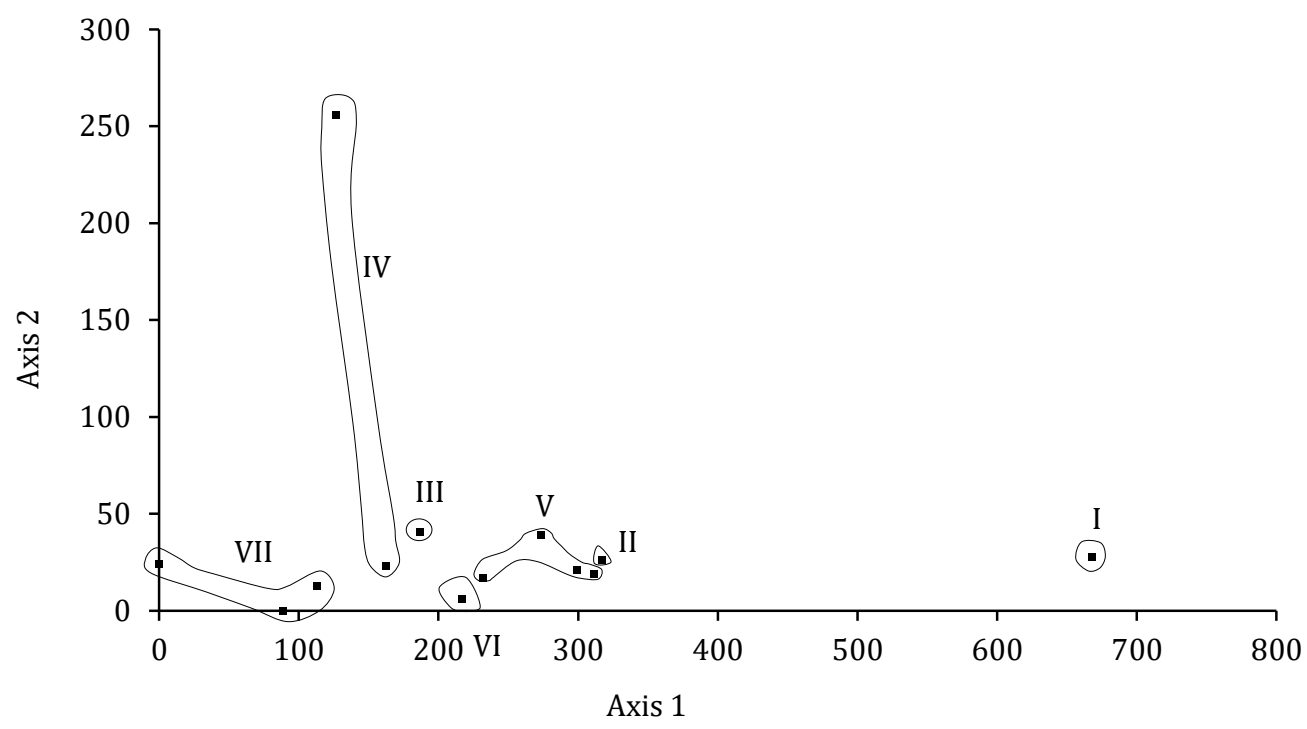

Fig. 3 - TWINSPAN classification (A) and DECORANA ordination (B) of the vegetation groups of the cultivated species associated with Moringa oleifera in 8 studied sites in Egypt based on their floristic composition. The 7 vegetation groups of the 13 sampled stands are encircled together (I - VII).

consisted of 3 stands (Sheikh Zuweid, Faculty of Science - Alexandria University and Faculty of Education - Ain Shams University) and dominated by Ceiba speciosa. It was clear that group VII had the highest number of species $(89$ species $=$
$51.1 \%$ of the total number of cultivated species), followed by groups II and III (each contained 45 species $=25.9 \%$ ), whereas group I contained the lowest number of species $(11$ species $=6.3 \%)($ Table 5$)$.

Table 5 - Characteristics of the 7 vegetation groups (I-VII) at level 5 after the application of TWINSPAN on the 13 sampled stands of cultivated species associated with Moringa oleifera in 8 studied sites in Egypt. P: Presence, C: Cover, VG: Vegetation group, NS: No. of stands, TS: total species.

\begin{tabular}{|c|c|c|l|r|r|l|c|r|}
\hline VG & NS & TS & First dominant species & $\begin{array}{c}\mathrm{C} \\
\%\end{array}$ & $\begin{array}{c}\mathrm{P} \\
\%\end{array}$ & Second dominant species & $\begin{array}{c}\mathrm{C} \\
\%\end{array}$ & $\begin{array}{c}\mathrm{P} \\
\%\end{array}$ \\
\hline I & 1 & 11 & Ficus elastica & 4 & 100 & Leucaena leucocephala & 3 & 100 \\
II & 1 & 63 & Abutilon hirtum & 7 & 100 & Quercus robur & 6 & 100 \\
III & 1 & 45 & Cordia myxa & 7 & 100 & Carya illinoensis & 5 & 100 \\
IV & 2 & 45 & Carya illinoensis & 8 & 50 & Ficus elastic & 5 & 50 \\
V & 4 & 14 & Azadirachta indica & 9 & 25 & Dendrocalamus giganteus & 7 & 25 \\
VI & 1 & 18 & Ficus benjamina & 5 & 100 & Leucaena leucocephala & 3 & 100 \\
VII & 3 & 89 & Ceiba speciosa & 10 & 33 & Carya illinoensis & 6 & 33 \\
\hline
\end{tabular}

2. Wild Species

\section{1. Taxonomic diversity}

The wild species associated with $M$. oleifera were 45 , related to 39 genera and 21 families (Table 6, Fig. 4). Dicotyledonous families were 17 represented by 29 genera and 34 species; the highly represented of 


\section{Kamal H. Shaltout et al.}

them were Asteraceae and Amaranthaceae (each of 7 species), Euphorbiaceae (4 species) and Solanaceae (3 species). Thirteen dicotyledonous families were represented only by one species. Monocotyledonous families were 3; the most represented was Poaceae (8 species), while each of Amaryllidaceae and Cyperaceae were represented by only one species. Pteridophytes were represented only by one species related to Pteridaceae. Portulaca oleracea was the most common associated wild species (presence $>40 \%$ ), followed by Bidens pilosa, Digitaria ciliaris, Oxalis corniculata, Cyperus rotundus, Lantana camara and Setaria viridis.

Table 6 - Wild species associated with Moringa oleifera in the 12 studied stands in Egypt. P: presence and $\mathrm{C}$ : cover. Life forms are coded as follows: $\mathrm{Ph}$ : phanerophytes; $\mathrm{Ch}$ : chamaephytes; Hc: hemicryptophytes; Ge: geophytes; Th: therophytes.

\begin{tabular}{|c|c|c|c|c|}
\hline \multirow{2}{*}{ Species } & \multirow{2}{*}{ Family } & \multirow{2}{*}{$\begin{array}{l}\text { Life } \\
\text { form }\end{array}$} & $\mathrm{P}$ & $\mathrm{C}$ \\
\hline & & & \multicolumn{2}{|c|}{$(\%)$} \\
\hline Adiantum capillus-veneris $\mathrm{L}$. & Pteridaceae & $\mathrm{Hc}$ & 8.3 & 0.5 \\
\hline Amaranthus viridis L. & Amaranthaceae & $\mathrm{Th}$ & 8.3 & 1.0 \\
\hline A.blitum L. & Amaranthaceae & $\mathrm{Th}$ & 25.0 & 2.1 \\
\hline A.hybridus L. & Amaranthaceae & $\mathrm{Th}$ & 8.3 & 1.0 \\
\hline A.retroflexus L. & Amaranthaceae & $\mathrm{Th}$ & 8.3 & 1.0 \\
\hline Bidens pilosa $\mathrm{L}$. & Asteraceae & $\mathrm{Th}$ & 50.0 & 20.0 \\
\hline Cenchrus echinatus L. & Poaceae & $\mathrm{Th}$ & 8.3 & 0.5 \\
\hline Centaurea pallescens Delile & Asteraceae & $\mathrm{Hc}$ & 8.3 & 0.1 \\
\hline Chenopodium album $\mathrm{L}$. & Chenopodiceae & $\mathrm{Th}$ & 8.3 & 0.1 \\
\hline C.murale L. & Chenopodiceae & $\mathrm{Th}$ & 16.7 & 2.0 \\
\hline Chichorium intybus L. & Asteraceae & $\mathrm{Th}$ & 8.3 & 1.0 \\
\hline Convolvulus arvensis L. & Convolvulaceae & $\mathrm{Ge}$ & 25.0 & 1.2 \\
\hline Crepis micrantha Czerep. & Asteraceae & $\mathrm{Th}$ & 8.3 & 1.0 \\
\hline Cynanchum acutum $\mathrm{L}$. & Apocynaceae & $\mathrm{Ph}$ & 8.3 & 1.0 \\
\hline Cynodon dactylon (L.) Pres. & Poaceae & $\mathrm{Ge}$ & 25.0 & 2.2 \\
\hline Cyperus rotundus L. & Cyperaceae & $\mathrm{Ge}$ & 41.1 & 7.5 \\
\hline Dactyloctenium aegptium (L.) Willd. & Poaceae & $\mathrm{Th}$ & 33.3 & 2.6 \\
\hline Datura innoxia Mill. & Solanaceae & Th & 16.7 & 2.0 \\
\hline Digitaria ciliaris (Retz.) Koeler & Poaceae & $\mathrm{Th}$ & 50.0 & 7.5 \\
\hline $\begin{array}{l}\text { Dysphania ambrosioides (L.) Mosyakin \& } \\
\text { Clemants }\end{array}$ & Amaranthaceae & Th & 25.0 & 2.2 \\
\hline Echinochloa colona (L.) Link & Poaceae & $\mathrm{Th}$ & 8.3 & 1.0 \\
\hline Erigeron bonariensis L. & Asteraceae & $\mathrm{Th}$ & 33.3 & 4.0 \\
\hline Euphorbia heterophylla L. & Euphorbiaceae & $\mathrm{Th}$ & 8.3 & 1.0 \\
\hline E.hirta L. & Euphorbiaceae & $\mathrm{Th}$ & 25.0 & 2.1 \\
\hline E.prostrata Aiton & Euphorbiaceae & $\mathrm{Th}$ & 16.7 & 4.0 \\
\hline Foeniculum vulgare Mill. & Apiaceae & $\mathrm{Hc}$ & 8.3 & 1.0 \\
\hline Heliotropium supinum L. & Boraginaceae & $\mathrm{Ch}$ & 8.3 & 0.1 \\
\hline Lantana camara $\mathrm{L}$. & Verbenaceae & $\mathrm{Ph}$ & 41.1 & 16.0 \\
\hline Malva parviflora $\mathrm{L}$. & Malvaceae & $\mathrm{Th}$ & 16.7 & 1.1 \\
\hline Oxalis corniculata $\mathrm{L}$. & Oxalidaceae & $\mathrm{Ge}$ & 50.0 & 7.1 \\
\hline Pancratium maritimum $\mathrm{L}$. & Amaryllidaceae & $\mathrm{Ge}$ & 16.7 & 4.0 \\
\hline Phytolacca americana $\mathrm{L}$. & Phytolaccaceae & $\mathrm{Hc}$ & 8.3 & 1.0 \\
\hline Plantago major L. & Plantaginaceae & $\mathrm{Hc}$ & 16.7 & 3.0 \\
\hline Portulaca oleracea L. & Portulacaceae & Th & 66.7 & 7.4 \\
\hline
\end{tabular}


Kamal H. Shaltout et al.

Table 6 - cont. 1

\begin{tabular}{|c|c|c|c|c|}
\hline \multirow{2}{*}{ Species } & \multirow{2}{*}{ Family } & \multirow{2}{*}{$\begin{array}{l}\text { Life } \\
\text { form }\end{array}$} & $\mathrm{P}$ & $\mathrm{C}$ \\
\hline & & & \multicolumn{2}{|c|}{$(\%)$} \\
\hline Ricinus communis L. & Euphorbiaceae & $\mathrm{Ph}$ & 33.3 & 4.1 \\
\hline Salsola kali L. & Chenopodiceae & Th & 8.3 & 0.1 \\
\hline Setaria verticillata (L.) P. Beauv. & Poaceae & Th & 33.3 & 4.6 \\
\hline S.viridis (L.) P.Beauv. & Poaceae & Th & 41.1 & 7.0 \\
\hline Solanum americanum Mill. & Solanaceae & $\mathrm{Ch}$ & 25.0 & 3.0 \\
\hline Sonchus oleraceus (L.) L. & Compositae & Th & 16.7 & 2.0 \\
\hline Sorghum virgatum (Hack.) Stapf & Poaceae & Th & 8.3 & 1.0 \\
\hline Trianthema portulacastrum L. & Aizoaceae & Th & 16.7 & 2.0 \\
\hline Tribulus pentandrus Forssk. & Zygophyllaceae & $\mathrm{Hc}$ & 16.7 & 0.6 \\
\hline $\begin{array}{l}\text { Verbesina encelioides (Cav.) Benth. \& Hook. } \\
\text { f. ex A. Gray }\end{array}$ & Compositae & $\mathrm{Th}$ & 8.3 & 1.0 \\
\hline Withania somnifera (L.) Dunal & Solanaceae & $\mathrm{Ch}$ & 8.3 & 1.0 \\
\hline
\end{tabular}

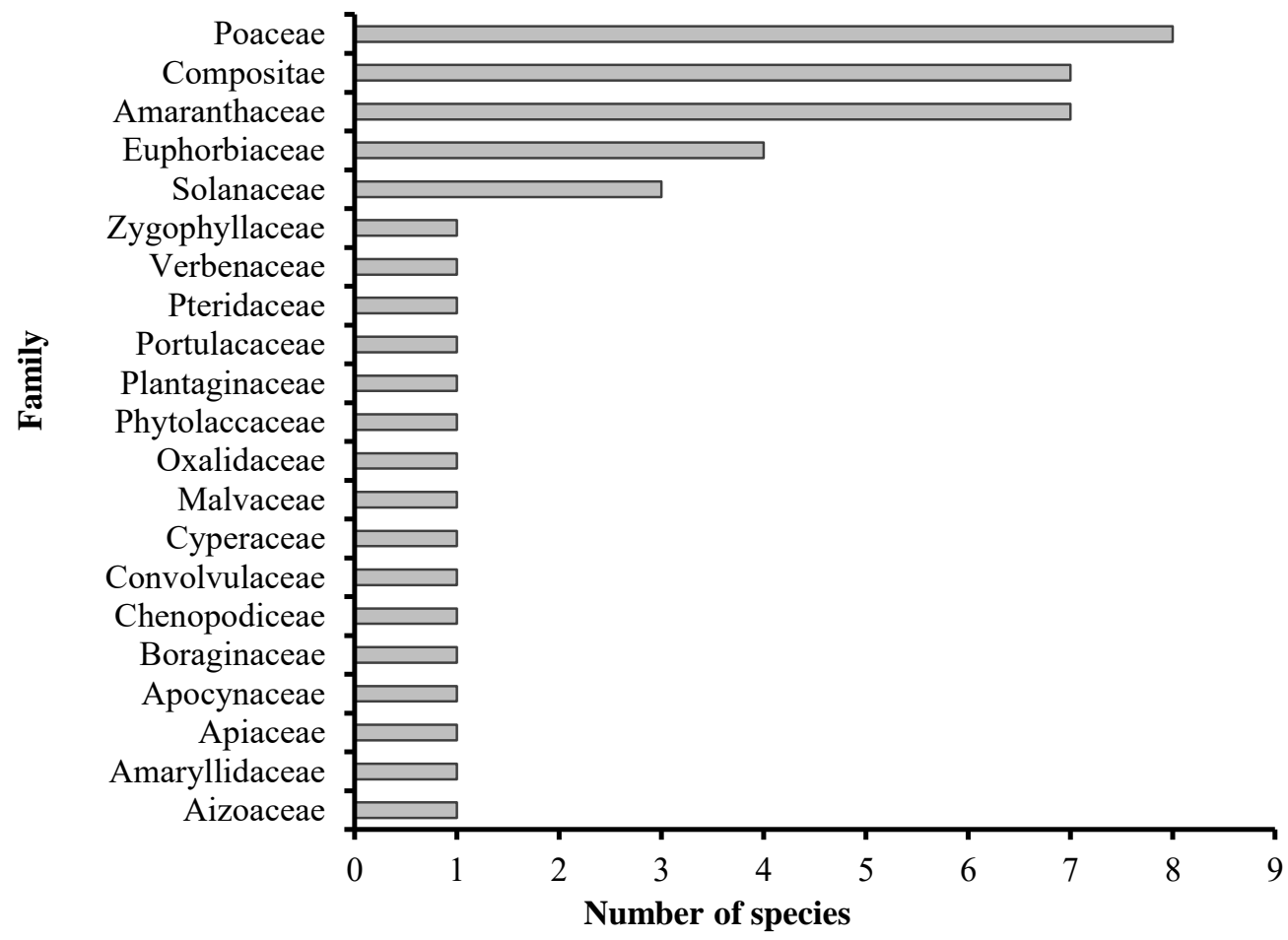

Fig. 4 - Number of the wild species associated with Moringa oleifera in relation to their families.

The taxonomic diversity of the 8 studied sites indicated that Qanatir Horticulture Research Institute had the maximum total number of species, genera and families. On the other hand, Antoniadis Garden had the minimum number of families, genera and species. Pteridophytes were represented only in Faculty of Science Alexandria University. The maximum number of dicotyledonous families, genera and species was recorded in Qanatir Horticulture Research Institute; while the maximum number of monocotyledonous families and species was recorded in Sheikh Zuweid (Table 7). 


\section{Kamal H. Shaltout et al.}

Table 7 - Taxonomic diversity of the wild species associated with Moringa oleifera in 7 studied sites in Egypt: Sheikh Zuweid Station (SZ), Botanic Garden of Faculty of Science Alexandria University (XS), Antoniadis Botanic Garden (XA), Qanatir Horticulture Research Institute (QA), Botanic Garden of Faculty of Science - Ain Shams University (SA), Botanic Garden of Faculty of Education - Ain Shams University (ED) and Orman Botanic Garden (OR).

\begin{tabular}{|l|rrrrrrr|r|}
\hline \multirow{2}{*}{ Taxic variable } & \multicolumn{7}{|c|}{ Study site } & \multirow{2}{*}{ Total } \\
\cline { 2 - 8 } & SZ & XS & XA & QA & SA & ED & OR & \\
\hline Dicotyledons & 7 & 7 & 4 & 24 & 12 & 7 & 7 & 34 \\
Species & 7 & 7 & 4 & 21 & 12 & 6 & 7 & 29 \\
Genus & 6 & 6 & 3 & 13 & 8 & 5 & 5 & 17 \\
Momily & & & & & & & & \\
Species & 6 & 2 & 3 & 4 & 5 & 2 & 4 & 10 \\
Genus & 2 & 2 & 3 & 4 & 5 & 2 & 4 & 9 \\
Family & 3 & 2 & 1 & 1 & 2 & 2 & 2 & 3 \\
Pteridophytes & & & & & & & & \\
Species & - & 1 & - & - & - & - & - & 1 \\
Genus & - & 1 & - & - & - & - & - & 1 \\
Family & - & 1 & - & - & - & - & - & 1 \\
\hline Total & & & & & & & & \\
Species & 13 & 10 & 7 & 28 & 17 & 9 & 11 & 45 \\
Genera & 13 & 10 & 7 & 25 & 17 & 8 & 11 & 39 \\
Families & 9 & 9 & 4 & 14 & 10 & 7 & 7 & 21 \\
\hline
\end{tabular}

\section{2. Life forms}

Determination of the life forms of the wild species associated with $M$. oleifera indicated that the therophytes was the most represented life form $(28$ species $=62.2 \%$ of the total species), followed by hemicryptophytes $(6$ species $=13.3 \%$ of the total species) and geophytes (5 species = $11.1 \%$ of the total species). Phanerophytes and chamaephytes were the least recorded life forms (each comprised 3 species $=6.7 \%$

of the total species) (Fig. 5). Regarding the flora of the 8 studied sites, the maximum representation of the chamaephytes, hemicryptophytes and therophytes was observed in Qanatir Horticulture Research Institute. On the other hand, the maximum representation of the phanerophytes was in Faculty of Science - Ain Shams University, while that of the geophytes was in Sheikh Zuweid (Table 8).

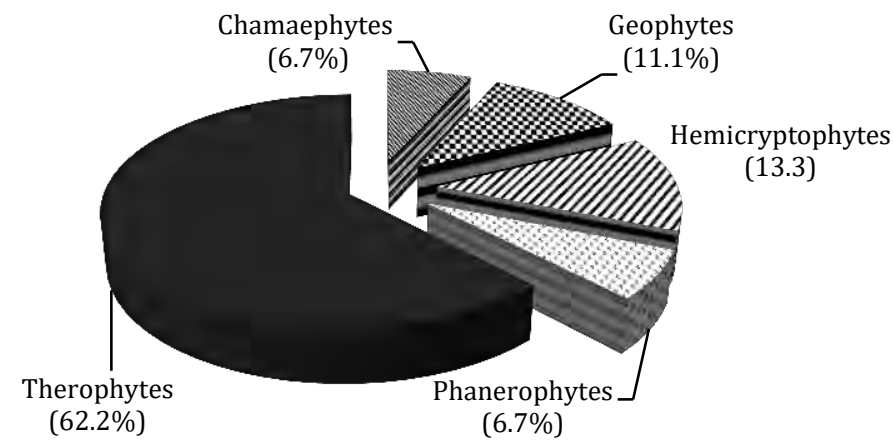

Fig. 5 - Life form spectrum of the wild species associated with Moringa oleifera in 7 studied sites in Egypt. 


\section{Kamal H. Shaltout et al.}

Table 8 - Life form spectrum of the wild species associated with Moringa oleifera in 7 studied sites in Egypt: Sheikh Zuweid Station (SZ), Botanic Garden of Faculty of Science Alexandria University (XS), Antoniadis Botanic Garden (XA), Qanatir Horticulture Research Institute (QA), Botanic Garden of Faculty of Science - Ain Shams University (SA), Botanic Garden of Faculty of Education - Ain Shams University (ED) and Orman Botanic Garden (OR).

\begin{tabular}{|l|ccccccc|c|}
\hline \multirow{2}{*}{ Life form } & \multicolumn{7}{|c|}{ Study site } & \multirow{2}{*}{ Total } \\
\cline { 2 - 8 } & SZ & XS & XA & QA & SA & ED & OR & \\
\hline Phanerophytes & - & - & 1 & 2 & 3 & 1 & - & 3 \\
Chamaephytes & - & - & 1 & 2 & 1 & - & - & 3 \\
Hemicryptophytes & 2 & 1 & - & 3 & 1 & - & - & 6 \\
Geophytes & 4 & 3 & - & 2 & 3 & 2 & 2 & 5 \\
Therophytes & 7 & 6 & 5 & 19 & 9 & 6 & 9 & 28 \\
\hline Total & 13 & 10 & 7 & 28 & 17 & 9 & 11 & 45 \\
\hline
\end{tabular}

\section{3. Vegetation analysis}

The application of TWINSPAN classification on the floristic composition of the 13 sampled stands led to classify them into 5 vegetation groups at level 3 (Fig. 6 A). The application of DECORANA on the same set of data indicates a reasonable segregation among these groups along the ordination axes 1 and 2 (Fig. 6 B). Group III included 1 stand (Faculty of Science - Alexandria University) and dominated by Cyperus rotundus, group I included 2 stands (Sheikh Zuweid) and dominated by Pancratium maritimum, group $\mathbf{V}$ represented by 2 stands (Qanatir Horticulture Research Institute and
Faculty of Education - Ain Shams University) and dominated by Bidens pilosa, group IV included 3 stands (Faculty of Science - Ain Shams University) and dominated by Lantana camara and group II comprised 4 stands (tow in Sheikh Zuweid, Antoniadis Garden and Orman Botanic Garden) and dominated by Digitaria ciliaris. It was clear that group $\mathbf{V}$ had the highest number of species ( 31 species $=68.9 \%$ of the total number of wild species), followed by group II (19 species $=42.2 \%)$, whereas group I contained the lowest number (7 species $=15.6 \%) \quad($ Table 9$)$.

\section{A- TWINSPAN classification}

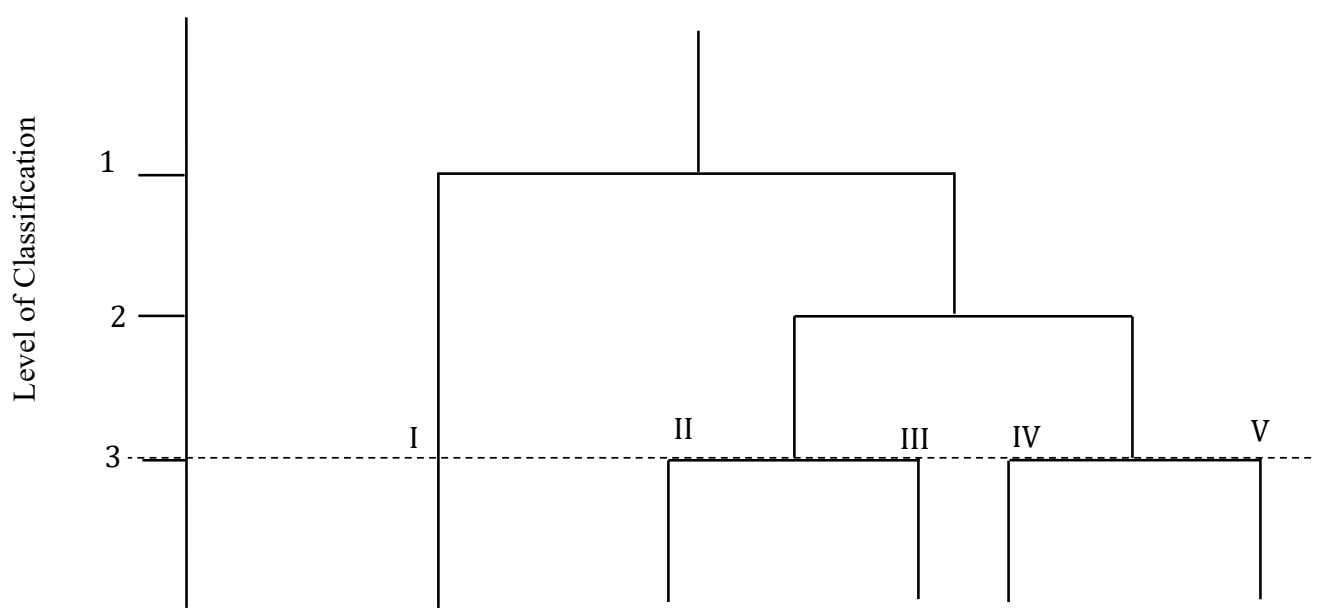




\section{Kamal H. Shaltout et al.}

B- DECORANA ordination

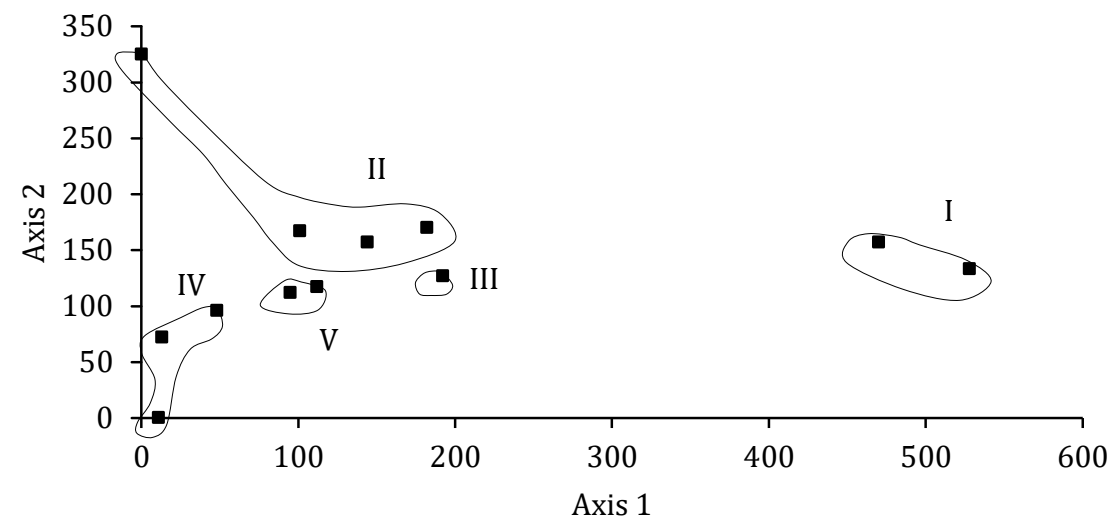

Fig. 6 - TWINSPAN classification (A) and DECORANA ordination (B) of the vegetation groups of the wild species associated with Moringa oleifera in 7 studied sites in Egypt based on their floristic composition. The 5 vegetation groups of the 12 sampled stands are encircled together $(\mathrm{I}-\mathrm{V})$.

Table 9 - Characteristics of the 5 vegetation groups (1-v) at level 3 after the application of TWINSPAN on the 12 sampled stands of wild species associated with Moringa oleifera in Egypt. P: Presence, C: Cover, VG: Vegetation group, NS: No. of stands, TS: Total species.

\begin{tabular}{|c|c|c|l|r|r|l|l|l|}
\hline VG & NS & TS & First dominant species & $\begin{array}{c}\text { C } \\
\%\end{array}$ & $\begin{array}{c}\text { P } \\
\%\end{array}$ & Second dominant species & $\begin{array}{c}\text { C } \\
\%\end{array}$ & $\begin{array}{c}\text { P } \\
\%\end{array}$ \\
\hline I & 2 & 8 & Pancratium maritimum & 4 & 100 & Verbesina encelioides & 1 & 50 \\
II & 4 & 28 & Digitaria ciliaris & 3 & 50 & Setaria verticillata & 2 & 75 \\
III & 1 & 10 & Cyperus rotundus & 2 & 100 & Euphorbia prostrata & 1 & 100 \\
IV & 3 & 29 & Lantana camara & 10 & 100 & Bidens pilosa & 8 & 100 \\
V & 2 & 37 & Bidens pilosa & 9 & 50 & Lantana camara & 6 & 100 \\
\hline
\end{tabular}

\section{Discussion}

The most represented family of the cultivated species associated with $M$. oleifera was Fabaceae, which is the third-largest terrestrial plant family in terms of number of species, after Orchidaceae and Asteraceae. It has 630 genera and over 18,860 species (Judd et al., 2002; Stevens, 2006). This family have a universal distribution, being found everywhere except Antarctica and the high arctic (Stevens, 2006). The cosmopolitan distribution of this family may be related to its wide variation of growth forms which includes trees, shrubs and herbaceous plants (annuals, biennials or perennials). They also could be upright plants, epiphytes or even vines. Also, they could be helophytes, mesophytes or xerophytes (Watson \& Dallwitz, 1992; Judd et al., 2002). In addition, many legumes are able to survive and compete effectively in nitrogen poor conditions (ildis.org) through a symbiotic relationship with some species of Rhizobium bacteria that present in root nodules. Many leguminous species, recorded in the present study, have medicinal importance as Cassia fistula (Danish et al., 2011) and Dichrostachys cinerea (Banso \& Adeyemo, 2007). Other species have been used as ornamentals throughout the world. The vast diversity of heights, shapes, foliage and flower colors caused this family to be commonly used in the design and planting of small gardens and even large parks (Burkart, 1987). Examples of these ornamentals which were recorded in the present study are Bauhinia variegata, Caesalpinia palmeri, Erythrina caffra, Erythrina corallodendrum 


\section{Kamal H. Shaltout et al.}

and Sophora secundiflora. On the other hand, Acacia farnesiana flowers contain an essential oil which was widely used in European perfumery (Duke, 1983). The valuable Leucaena leucocephala produces a large volume of medium-light hardwood for fuel (specific gravity of $0.50-0.75$ ) with low moisture and high heating value, and makes an excellent charcoal that yields little ash and smoke. It also can be used for flooring, small furniture as well as for paper pulp production (Shelton \& Brewbaker, 1998).

The most represented life form was the phanerophytes, which had a superior representation in botanic gardens of Faculties of Science and Education (Ain Shams University), then Botanic Garden of Faculty of Science - Alexandria University. Phanerophytes are dominant in tropical regions of the world, even that, as recorded in this study, they can be recorded in sites other than the tropics. This may be attributed to their adaptability to life in less favorable localities by the protection of the growing point against the effects of the unfavorable season through many efficient ways (Raunkiaer, 1934). Some deciduous phanerophytes became adapted through leaf fall. Examples of this in the present study are Cordia myxa, Ginkgo biloba, Morus alba, Taxodium distichum, Vitex agnus-castus and Vitis vinifera. Other evergreen phanerophytes, like Ficus sp. had their buds being protected by unexpanded adherent stipules. Whereas other evergreens like Melaleuca sp., their new leaves become full grown and still folded for a long time, thus helping the enclosed younger leaves to achieve a considerable development before being subjected to direct air desiccation. Furthermore, depending on Raunkiaer classification of life forms (Raunkiaer, 1934), we can assume that, Pelarogonium zonale and Musa paradisiaca are examples of herbaceous phanerophytes, while Monstera deliciosa is an epiphytic phanerophyte and Cereus uruguayanus is a stem-succulent phanerophyte.

Phoenix dactylifera was the most common associated species with $M$. oleifera. Together with the olive, grape and fig, $P$. dactylifera were amongst the oldest cultivated plants of human kind and used as food for 6000 years (Zohary \& Speigel-Roy, 1975; Sulieman et al., 2012). P. dactylifera is well-suited to vegetative propagation because it produces offshoots at the trunk base which can be used in simple clonal reproduction of chosen palms (palmweb.org). This Plant can grow in very hot and dry climates, and can tolerate salty and alkaline soils. It requires a long, intensely hot summer with little precipitation and very low humidity during the stage from pollination to harvest, but with abundant underground water near the surface or irrigation. Such conditions are found in the oases and valleys in the arid sub-tropical deserts of the Middle East and North Africa countries (Jaradat, undated).In Egypt, It was recorded to be mainly distributed in the following habitats: along rail and high ways, abandoned fields and drains (Shaltout et al., 2010 b).

The wide distribution of $P$. dactylifera may be related to its commercial importance. It is the main crop in Egypt, Saudi Arabia, and Middle East countries (Chao \& Krueger, 2007).The primary use of date palms is, of course, their nourishing fruit which is eaten fresh, dried or processed as one of a widerange of date products. Date fruits can be stored and transported easily and hence become an important component of the Middle East diets. In addition, its vegetative parts are used as building materials (leaves, trunks), fencing (leaves, midribs), thatch (leaves), rope (fibres of leaf sheath, leaflet and midrib), fuel (all vegetative parts, but especially leaf bases); packaging, padding and protection (leaf sheath fibre) (Barrow, 1998).

Numerous popular plants (e.g., $P$. dactylifera) as well as other rare species (e.g., Ginkgo biloba) were recorded in many botanic gardens. This confirms the important role of these gardens in conservation of threatened species. Amaglo (2006) stated that, to cultivate $M$. oleifera with other crops, it is important to choose species that are adapted to alley cropping, such as shadetolerant leafy vegetables, legumes and herbs. Many studies tried to determine the appropriate species which can be associated with $M$. oleifera and their suitable intercropping models (e.g., Swaminathan et 


\section{Kamal H. Shaltout et al.}

al., 1999; Palada et al., 2003; Shanmughavel et al., 2003; Immanuel \& Ganapathy, 2010).

The maximum number of species, genera and families was recorded in the Botanic Gardens, especially that of Faculty of Science - Ain Shams University. Most of these species were introduced from different countries and cultivated in the form of mixture of human-made microhabitats (e.g., shrub beds, rockeries, hedges, walls, flower beds and compost heaps), and being receiving large amounts of materials including nutrients (Gilbert, 1991). The most crowded botanic garden was that of Faculty of Education - Ain Shams University (Khalifa, 2006); where 300 species related to 70 families were housed in only $700 \mathrm{~m}^{2}$. Not only, had the valuable plants in this garden subjected to damage by crowdedness, but also to unwise practices like laborer shortage (author observation).According to the authors opinion, the best served and cared botanic garden was the Botanic Garden of Faculty of Science (Alexandria University) followed by Aswan Botanic Garden.

Classification and ordination of the sample stands of the cultivated species in the present study indicated a clear distinction of seven groups which were recognized on the basis of their characteristic species as follows: Ficus elastica - Leucaena leucocephala, Abutilon hirtum - Quercus robur, Cordia myxa - Carya illinoensis, Carya illinoensis - Ficus elastica, Azadirachta indica- Dendrocalamus giganteus, Ficus benjamina - Leucaena leucocephala and Ceiba speciosa - Carya illinoensis. It was observed that Ficus elastica was the $1^{\text {st }}$ dominant species in group I and the $2^{\text {nd }}$ in group III. Both groups were related to botanic gardens which were characterized by presence of shade tolerant species like $F$. elastica (cabi.org). $F$. elastica thrives in moister, warmer, tropical climates and in vegetation zones of tropical rain forest, wood and shrub lands and light tropical forest, but apparently can tolerate temperature range of $0-10{ }^{\circ} \mathrm{C}$ in winter and $10-30{ }^{\circ} \mathrm{C}$ in summer, with an annual precipitation up to $200 \mathrm{~mm}$ (Starr et al., 2003). This species tolerates drought, and can be grown in the well-drained soil (Gilman \& Watson, 2014).
It can reproduce by both cuttings and seeds (Starr et al., 2003; Gilman \& Watson, 2014). Its root system is shallow and dense, making mixed plantation or intercropping systems unfeasible (proseanet.org). In the $19^{\text {th }}$ and $20^{\text {th }}$ centuries, it was heavily cultivated to produce commercial rubber, but fell into disuse with the rise of production of the higher-quality rubber from Hevea brasiliensis (Tawan, 2000; Whistler, 2000).

Carya illinoensis was the $1^{\text {st }}$ dominant species in group IV and the $2^{\text {nd }}$ in groups III and VII. These groups characterized the Botanic Gardens of Faculties of Science and Education of Ain Shams University. It is a long-lived, medium to large, deciduous tree ranging from 30 to $45 \mathrm{~m}$ in height and 1.8 to $2.1 \mathrm{~m}$ in diameter (Duncan \& Duncan, 1988). It is widely planted as an ornamental, and for its sweet edible nuts which are used widely in candies and cookies (Stephens, 1973). This tree prefers well-drained loamy soils not subjected to prolonged flooding (Peterson, 1990), and rarely grows on low and poorly drained clay flats (Allen \& Kennedy, 1989). It is shade intolerant (Peterson, 1990) with its deep lateral roots providing excellent watershed protection (Wasser, 1982).

Leucaena leucocephala was the $2^{\text {nd }}$ dominant species in groups I and VI. It is characterized by plentiful, precocious and year-round seed production (building up a substantial seed bank), lack of pollinator specificity, ability to renew after cutting or burning, drought tolerance, ability to produce thickets, and self-compatibility meaning that it can spread from an isolated tree (Hughes \& Jones, 1998). Once established, this species is difficult to eradicate, as the soil seed bank is able to remain viable for at least 10 years after removal of seeding trees (Walton, 2003). Despite preceding points, Parrotta (1993) mentioned that M. oleifera was found to grow in association with this species in the secondary forests (south coastal plain of Puerto Rico).

Regarding the wild flora associated with $M$. oleifera, the most represented family was Poaceae. It has grains of intermediate weight capable of immediate germination and rapid growth; and many of their species have ability for extensive lateral spread providing flexible regeneration strategies. These 


\section{Kamal H. Shaltout et al.}

characters equipped this family well to exploit man-made habitats (Hodgson, 1986; Gilbert, 1991). The absence of associated species in Aswan Botanic Garden may be due to the paved ground around $M$. oleifera trees; hence the weakly rooted species cannot penetrate it. In addition, the most recorded life form was the therophytes which had highest representation in Qanatir Horticulture Research Institute. Comparing with the perennial species, therophytes have higher reproductive capability and morphological, ecological and genetic plasticity under high levels of disturbances (Harper, 1977; Grime, 1979).

In the present study, Portulaca oleracea was the most common wild species associated with $M$. oleifera. Shaltout et al. $(2010$ b) had recorded this species to be distributed in numerous habitats in Nile Delta (e.g., high ways, waste lands, abandoned fields, fields of orchards, fields of summer and winter crops, canals and drains). In addition, Matthews et al. (1993) reported that $P$. oleracea is a weed of open, disturbed habitats and almost of global adaptability and distribution. The self-compatible breeding system, seed dormancy, and ability of seeds to resist animal digestive processes; facilitated to ensure its survival and distribution.

Classification and ordination of sampled stands of the wild species in the present study indicated a clear distinction of five groups which were recognized on the basis of their characteristic species as follows: Pancratium maritimum -Verbesina encelioides, Digitaria ciliaris- Setaria verticillata, Cyperus rotundus -Euphorbia prostrata, Lantana camara - Bidens pilosa and Bidens pilosa - Lantana camara. Pancratium maritimum was the $1^{\text {st }}$ dominant species in group I. It is an Amaryllidaceous endangered species (Grassi et al., 2005). In Egypt, it is distributed along the Mediterranean coastal region including Deltaic and Sinai coast (Shaltout et al., 2010 b; El-Hadidy et al., 2011). It can colonize sandy shores of different geologies and in some cases, rocky cliffs (Giovino et al., 2015). The salt stress tolerance of $P$. maritimum in maritime habitats is caused by proline production activity (Khedr et al.,
2003). In recent years, the level of attention given to this species has increased due to its importance as a bio-indicator, the potential industrial value of its chemical compounds, and its use as an ornamental plant (Abbassy et al., 1998; Sanaa et al., 2013).

Lantana camara was the $1^{\text {st }}$ dominant species in group IV and the $2^{\text {nd }}$ in group $\mathbf{V}$. It is a beautiful plant, often planted in gardens, and had been introduced from Central and South America to many countries like Egypt to be used as an ornamental plant (Day, 2003). Shaltout et al. (2016) recorded it as one of the naturalized species in many habitats in Nile region including waste places, fields, fence lines and pasture. The wide geographic distribution of lantana indicates its wide ecological tolerance. It occurs in diverse habitats and on different soil types. In general, it grows best in open un-shaded situations such as rainforest edges, beachfronts, wastelands, and forests recuperating from fire or logging. This species also favors disturbed sites such as road and railway sides and canal banks (Winder \& Harley, 1983; Thakur et al., 1992; Munir, 1996). L. camara is a major problem in agricultural areas in most countries in which it occurs (Holm et al.,1991), because it reduces the productivity in pasture by the formation of compact thickets which reduce growth of crops as well as make harvesting more difficult (Okoth, 1987).

Bidens pilosa was the $1^{\text {st }}$ dominant species in group $\mathbf{V}$ and the $2^{\text {nd }}$ in group $\mathbf{I V}$. It is a cosmopolitan, annual therophyte which originates from tropical and Central America (issg.org).High seed production and ability to grow in many habitats are the most important survival strategies (Souza et al., 2009). This species is capable of invading numerous habitats ranging from moist soil, sand, limerock, or dry, infertile soil and low to high elevations of up to $3600 \mathrm{~m}$. It grows well in disturbed areas, high sunlight, and moderately dry soils. It is known to invade grassland, forest clearings, wetlands, plantations, streamlines, canals, roadsides, rail ways, pasture, coastal areas and agriculture areas(PIER, 2007; DPI, 2008; Shaltout et al., 2010 b). The vast distribution of this species together with $P$. oleracea, $L$. camara, must be taken in consideration. 



\section{Kamal H. Shaltout et al.}

Protection and security plans, designed by the experts, should be applied in the sites where $M$. oleifera crop will be cultivated in Egypt to prevent the probable damage caused by these harmful weeds.

\section{Acknowledgement}

Special thanks are due to Dr. Dalia Abd ElAzeem Ahmed (Assistant Prof. of Plant Ecology - Botany Department - Faculty of Science - Tanta University) for her help in the multivariate analysis of the vegetation data.

\section{References}

Abbassy MA, El-Gougary OA, El-Hamady S, Sholom MA (1998). Insecticidal, acaricidal and synergistic effects of soosan, Pancratium maritimum extracts and constituents. Journal of the Egyptian Society of Paracitology 28:197-205.

Akinbode OA, Ikotun T (2008). Efficacy of certain plant extracts against seed-borne infection of Collectotrichum destructivum on cowpea (Vigna uniguculata). African Journal of Biotechnology 7(20):3683-3685.

Allen JA, Kennedy HE (1989). Bottom land hardwood reforestation in the lower Mississippi Valley. Slidell, LA: U.S. Department of the Interior, Fish and Wildlife Service, National Wetlands Research Center; Stoneville, MS: U.S. Department of Agriculture, Forest Service, Southern Forest Experimental Station. pp: 28.

Amaglo N (2006). How to Produce Moringa Leaves Efficiently? Moringa and other highly nutritious plant resources: Strategies, standards and markets for a better impact on nutrition in Africa. Accra, Ghana, November 16-18, 2006 Workshop 2. pp: 11.

Ammar EE (2015). Current situation of agro-biodiversity in Nile Delta. M.Sc. Thesis. Tanta University, Tanta. pp: 274.

Anwar F, Ashraf M, Bhanger MI (2005). Interprovenance variation in the composition of Moringa oleifera oilseeds from Pakistan. Journal of American Oil Chemists' Society 80(1):151-155.

APG II System (2003). APG is the latest Angiospermic Phylogenic Classification made by the APG (Angiospermic Phylogeny Group II System). [http://www.arsgrin.gov/ cgibin/npgs /html/inddex.pl.].
Bailey LH (1960). The Standard Cyclopedia of Horticulture. Vol. IV. New York, The Macmillan Company. pp: 2067-2068.

Banso A, Adeyemo SO (2007). Evaluation of antibacterial properties of tannins isolated from Dichrostachys cinerea. African Journal of Biotechnology 6(15): 1785-1787.

Barrow SC (1998). A Monograph of Phoenix L. (Palmae: Coryphoideae). Kew Bulletin 53(3):513-575.

Botanica (2004). The Illustrated A-Z of over 10.000 Garden Plants. KÖNEMANN, an imprint of Tandem Verlag $\mathrm{GmbH}$, Königswinter. pp: 1020.

Boulos L (1999). Flora of Egypt. Vol. I, (Azollaceae-Oxalidaceae). Al Hadara Publishing, Cairo, Egypt. pp: 419.

Boulos L (2000). Flora of Egypt. Vol. II, (Geraniaceae-Boraginaceae). Al Hadara Publishing, Cairo, Egypt. pp: 352.

Boulos L (2002). Flora of Egypt. Vol. III, (Verbenaceae-Compositae). Al Hadara Publishing, Cairo, Egypt. pp: 373.

Boulos L (2005). Flora of Egypt. Vol. IV, Monocotyledons (AlismataceaeOrchidaceae). Al Hadara Publishing, Cairo, Egypt. pp: 617.

Brandon N, Shelton HM (1993). The role of vesicular-arbuscular mycorrhizae in establishment of leucaena. Proceedings of XVII Grassland Congress, Australia. New Zealand Grassland Association, New Zealand. pp: 2064-2065.

Burkart A (1987). Leguminosas. In: Dimitri M. Enciclopedia Argentina de Agricultura y Jardinería. Tomo I. Descripción de plantas cultivadas. Editorial ACME S.A.C.I., Buenos Aires. pp: 467-538.

Chao CT, Krueger RR (2007). The date palm (Phoenix dactylifera L.): Overview of biology, uses, and cultivation. HortScience 42:1077-1082.

Danish M, Singh P, Mishra G, Srivastava S, Jha1 K, Khosa R (2011). Cassia fistula Linn. (Amulthus) - An important medicinal plant: A review of its traditional Uses, phytochemistry and Pharmacological Properties. Journal of Natural Product and Plant Resources 1(1):101-118.

Day MD (2003). Lantana: current management status and future prospects. Australian Centre for International Agricultural Research: Canberra. pp: 128. 


\section{Kamal H. Shaltout et al.}

[http://aciar.gov.au/publication/mn102]

DPI (Department of Primary Industries) (2008). Victorian Resources Online Statewide. Cobblers Pegs (Bidens pilosa L.): Impact Assessment - Cobblers Pegs (Bidens pilosa L.) in Victoria. [http://hear.org/starr /publications/2006_lanai_islets].

Duke JA (1983). Moringa oleifera Lam. Handbook of Energy Crops. [http://www.hort.purdue.edu/newcrop/duke_ energy/Moringa oleifera.html].

Duncan WH, Duncan MB (1988). Trees of the southeastern United States. Athens, GA: The University of Georgia Press. pp: 322.

El-Hadidy A, Abd El-Ghani M, Amer W, Hassan R (2011). Systematic Revision of the Genus Pancratium L. (Amaryllidaceae) in Egypt with a New Addition. Notulae Scientia Biologicae3:24-38.

Gilbert LO (1991). The Ecology of Urban Habitats. London: Chapman and Hall. Univ. Press, Cambridge. pp: 369.

Gilman EF, Watson DG (2014). Ficus elastica, Fact Sheet ENH 411. Florida, USA: Environmental Horticulture Department, Institute of Food and Agricultural Sciences (IFAS), University of Florida. [http://edis.ifas.ufl.edu/pdffiles/ST/ST25200. pdf].

Giovinoa A, Dominab G, Bazanc G, Campisic P, Scibettaa S (2015). Taxonomy and conservation of Pancratium maritimum (Amaryllidaceae) and relatives in the Central Mediterranean. Acta Botanica Gallica: Botany Letters pp: 11.

Grassi F,Cazzaniga E, Minuto L, Peccenini S, Barberis G, Basso B (2005). Evaluation of biodiversity and conservation strategies in Pancratium maritimum $\mathrm{L}$. for the Northern Tyrrhenian Sea. Biodiversity and Conservation 14(9):2159-2169.

Grime JP (1979). Plant Strategies and Vegetation Processes. London: J. Wiley. pp: 222.

Hamdy RS, Abd El-Ghani MM, Youssef TL, El-Sayed M (2007). The Floristic composition of some historical Botanical Gardens in the metropolitan of Cairo, Egypt. African Journal of Agricultural Research 2(11):610-648.

Harper JL (1977). Population Biology of Plants. London. Academic Press. pp: 892.

Heneidy SZ (2010). Plant Atlas - The
Botanic Garden (Alex), Faculty of Science, Alexandria University. pp: 632.

Hill MO (1979 a). TWINSPAN-A FORTRAN program for arranging multivariate data in an ordered two-way table by classification of the individuals and attributes. Cornell University, Ithaca. $52 \mathrm{pp}$.

Hill MO (1979 b). DECORANA-A FORTRAN program for detrended correspondence analysis and reciprocal averaging. Cornell University, Ithaca. $90 \mathrm{pp}$.

Hodgson JG (1986). Commonness and rarity in plants with special reference to the Sheffield flora. Parts I-IV. Conservation Biology 36:199-314.

Holm LG, Plucknett DL, Pancho JV, Herberger JP (1991). The World's Worst Weeds: Distribution and Biology. Krieger Publishing Company, Malabar, Florida. pp: 609.

Hughes CE, Jones RJ (1998). Environmental hazards of leucaena. To Leucaena: Adaptation, Quality and Farming Systems Workshop HM.

Immanuel RR, Ganapathy M (2010). Performance of field crops along with four tree species in coastal Tamil Nadu. Indian Journal of Forestry 33(1):67-70.

Iqbal S, Bhanger MI (2006). Effect of season and production location on antioxidant activity of Moringa oleifera leaves grown in Pakistan. Journal of Food Composition and Analysis 19 (6-7):544-551.

Judd WS, Campbell CS, Kellogg EA, Stevens PF, Donoghue MJ (2002). Plant systematics: A phylogenetic Approach, Sinauer Axxoc. pp:287-292.

Kajihara R, Nakatsu S, Shiono T, Shibata $\mathrm{K}$, Ishiahara M, Sakamoto $\mathrm{K}$, Muto $\mathbf{N}(2008)$. Antihypertensive effect of water extracts from leaves of Moringa oleifera Lam. on spontaneously hypertensive rats. Japanese Society of Food and Technology (Nihon Shokuhin Kogyo Gakkai), Tsukubagan, Japan, 55(4):183-185.

Khalifa SF, Loutfy MH (2006). Ornamental cultivated plant collection. First International Conference on Strategy of Botanic Gardens. Agriculture Museum, Dokki, Cairo.

Khedr AH, Abbas MA, Abdel Wahid AA, Quick WP, Abogadallah GM (2003). Proline induces the expression of salt-stressresponsive proteins and may improve the 


\section{Kamal H. Shaltout et al.}

adaptation of Pancratium maritimum L. to salt stress. Journal of Experimental Botany 54(392):2553-2562.

Matthews JF, Ketron DW, Zane SF (1993). The biology and taxonomy of the Portulaca oleracea L. (Portulacaceae) complex in North America. Rhodora 95:166183.

Mohan J, Sharma S, Mahida Y, Ishnava K (2006). Spectrum of antibacterial activity possessed by petal extracts of some Indian plants. Journal of Medicinal and Aromatic PlantSciences 28(4):557-561.

Muenscher WC (1980). Weeds. $2^{\text {nd }}$ ed. Comstock Publishing Assoc. Cornell University Press. Ithaca, NY.

Muller-Dombois D, Ellenberg H (1974). Aims and Methods of Vegetation Ecology. New York, John Wiley \& Sons. pp:547.

Munir AA (1996). A taxonomic review of Lantana camara L. and L. montevidensis (Spreng.) Briq. (Verbenaceae) in Australia. Journal of the Adelaide Botanical Gardens 17:1-27.

Mvere B (2004). Bidens pilosa L. In: Grubben, G. J. H. and Denton, O. A. (Editors). Prota 2: Vegetables/Legumes. [CDRom]. Prota, Wageningen, Netherlands. [http://database.prota.org/PROTAhtml/Biden -s\%20pilosa_En. htm].

Okoth JO (1987). A study of the resting sites of Glossina fuscipes (Newstead) in relation to Lantana camara thickets and coffee and banana plantations in the sleeping sickness epidemic focus, Busoga, Uganda. Uganda Trypanosomiasis Research Organization 8:57-60.

Palada MC, Chang LC (2003). Suggested cultural practices for Moringa. International Cooperators' Guide, March. pp: 5.

Palada MC, Becker BN, Mitchell JM, Nair PK (2003). Cultivation of Medicinal Plants in Alley Cropping System with Moringa oleifera in the Virgin Islands. In: Advancing Caribbean Herbs in the 21st Century. Proceedings of the Sixth International Workshop on Herbal Medicine in the Caribbean hosted by the Caribbean Association of Researchers and Herbl Practitioners, June 27-29, 2003.Edited by Yuri N. Clement and Compton E. Seaforth. MPC Printers, UWI, St. Augustine, Trinidad and Tobago. pp: 60-83.
Pandey R, Kalra A, Katiyar N, Kumar S (2001). Nematicidal activity in flowers of some medicinal and aromatic plants. Indian Journal of Nematology 31(1):96-98.

Parrotta JA (1993). Moringa oleifera Lam. Reseda, horseradish tree. Moringaceae. Horseradish tree family. USDA Forest Service, International Institute of Tropical Forestry. pp: 6.

Peterson JK (1990). Carya illinoensis (Wangenh.) K. Koch pecan. In: Burns, Russell M, Honkala, Barbara H., technical coordinators. Silvics of North America. Vol. II. Hardwoods. Agriculture Handbook. 654. Washington, DC: U.S. Department of Agriculture, Forest Service. pp:205-210.

PIER (Pacific Island Ecosystems at Risk) (2007). Bidens pilosa L., Asteraceae [http://www.hear.org/pier/species/bidenspilosa.htm].

RHS (The New Royal Horticultural Society) (1992). Dictionary of Gardening. (ed. Huxley A.). Publisher Macmillon, UK, Four volumes. pp: 3239.

Raunkiaer C (1934). Plant Life Forms. Clarendon Press, Oxford. pp: 104.

Sanaa A, Boulila A, Boussaid M, Fadhel N (2013). Alginic acid and derivatives, new polymers from the endangered Pancratium maritimum L. Industrial Crops and Products 44:290-293.

Shaltout KH, Hosni HA, El-Kady HF, ElBehery MA, Shaltout SK (2016). Composition and pattern of alien species in the Egyptian flora. Flora 222:104-110.

Shaltout K, Shraf El-Din A, Ahmed D (2010). Plant life in the Nile Delta. Tanta University Press. pp: 232.

Shanmughavel P, Paddappaiah RS, Francis K (2003). Experimental testing of two agri-silvi-cultural models for semi-arid regions.Journal of Sustainable Forestry 17(4):91-98.

Shelton HM, Brewbaker JL (1998). Forage Tree Legumes in Tropical Agriculture. Leucaena leucocephala - the most widely used forage tree legume. [http://www.fao.org/ ag/agp/agpc/doc/publicat/gutt-shel/x5556e06. htm].

Singh KA (2001). Leaf morphology and leaf area of fodder trees of NEH region. RangeManagement and Agroforestry 22(1):85-93. 


\section{Kamal H. Shaltout et al.}

Souza MC, Pitelli RA, Simi LD, Oliveira MC (2009). Seed emergence of Bidens pilosa at different sowing depths. Planta Paninha 27(1):29-34.

Starr F, Starr K, Loope L (2003). Ficus elastica: Indian rubber tree. Hawaii, USA: United States Geological Survey. [http://www.hear.org/starr/hiplants/reports/pd f/ficus_elastica.pdf].

Stephens HA (1973). Woody plants of the North Central Plains. Lawrence, KS: The University Press of Kansas. pp: 530.

Stevens PF (2006). Fabaceae. Angiosperm Phylogeny Website. [http://www. mobot.org /mobot/research/apweb/orders/fabalesweb.ht $\mathrm{m} \#$ fabaceae].

Sulieman A, Elhafise I and Abdelrahim A (2012). Comparative study on five Sudanese date (Phoenix dactylifera L.) fruit cultivars. Food and Nutrition Sciences 3:1245-1251.

Swaminathan C, Robin S, Kannan K, Malarvizhi D and Dhanakodi C V (1999). Effects of Eucalyptus and Casuarina on the yield of intercrops in agroforestry systems. Allelopathy Journal 6(2):251-260.

Täckholm V (1974). Students' Flora of Egypt: $2^{\text {nd }}$ ed. Cairo University Press, Cairo. pp: 888.

Tawan C (2000). Ficus elastica Roxb. In: Plant Resources of South-East Asia (Prosea) No. 18: Plants Producing Exudates [ed. by Boer E, Ella A]. Leiden, The Netherlands: Backhuys Publisher. pp: 69-73.

Thakur ML, Ahmad M, Thakur RK (1992). Lantana weed (Lantana camara var. aculeata Linn) and its possible management through natural insect pests in India. Indian
Forester 118:466-488.

Thaman RR (1974). Lantana camara: Its introduction, dispersal and impact on islands of the tropical Pacific Ocean. Micronesica 10:17-39.

The Plant List (2013). [http://www. theplantlist.org/].

Virmani M, Garg S (2005). In vitro antiviral activity of plant extracts against equine herpes virus-1. Indian Journal of Comparative Microbiology, Immunology and Infectious Diseases 26(2):89-91.

Walton CS (2003). Leucaena (Leucaena leucocephala) in Queensland. Pest status review series - land protection. Department of Natural Resources and Mines, Qld.

Wasser CH (1982). Ecology and culture of selected species useful in revegetating disturbed lands in the West. FWS/OBS82/56. Washington, DC: U.S. Department of the Interior, Fish and Wildlife Service. pp: 347.

Watson L, Dallwitz MJ (1992) onwards. The families of flowering plants: descriptions, illustrations, identification, and information retrieval. (delta-intkey.com).

Whistler WA (2000). Tropical ornamentals; A Gide. Portland, Oregon, USA: Timber Press.

Winder JA and Harley KL (1983). The phytophagous insects on Lantana in Brazil and their potential for biological control in Australia. Tropical Pest Management 29:346-362.

Zohary D and Spiegel-Roy P (1975). Beginning of fruit growing in the Old World. Science 187:319-327. 